\title{
POLYCHAETE ASSEMBLAGE OF AN IMPACTED ESTUARY, GUANABARA BAY, RIO DE JANEIRO, BRAZIL
}

\author{
Leonardo Santi ${ }^{I}$ and Marcos Tavares ${ }^{2}$ \\ ${ }^{1}$ Universidade Santa Úrsula, Instituto de Ciências Biológicas e Ambientais \\ (Rua Jornalista Orlando Dantas, 59, 22231-010 Rio de Janeiro, RJ, Brasil) \\ ${ }^{2}$ Museu de Zoologia da Universidade de São Paulo \\ (Avenida Nazaré, 481, 04263-000, São Paulo, SP, Brasil) \\ mdst@usp.br
}

\section{A B S T R A C T}

Thirty-eight stations were sampled in Guanabara Bay, Rio de Janeiro, Brazil, to assess the spatio-temporal diversity and biomass of sublittoral polychaetes. Samples were collected during the dry (September 2000) and rainy season (May 2001) in shallow sublittoral sediments. The polychaete spatial composition showed a heterogeneous distribution throughout the bay. A negative gradient of diversity and biomass was observed towards the inner parts of the bay and sheltered areas. A wide azoic area was found inside the bay. Some high-biomass and low-diversity spots were found near a sewage-discharge point. In these areas, the polychaete biomass increased after the rainy season. A diversified polychaete community was identified around the bay mouth, with no dramatic changes of this pattern between the two sampling periods. Deposit-feeders were dominant in the entire study area. The relative importance of carnivores and omnivores increased towards the outer sector, at stations with coarse sediment fractions. Guanabara Bay can be divided into three main zones with respect to environmental conditions and polychaete diversity and biomass patterns: A) High polychaete diversity, hydrodynamically exposed areas composed of sandy, oxidized or moderately reduced sediments with normoxic conditions in the water column. B) Low diversity and high biomass of deposit and suspension-feeding polychaete species in the middle part of the bay near continental inflows, comprising stations sharing similar proportions of silt, clay and fine sands. C) Azoic area or an impoverished polychaete community in hydrodynamically low-energy areas of silt and clay with extremely reduced sediments, high total organic matter content and hypoxic conditions in the water column, located essentially from the mid-bay towards the north sector. High total organic matter content and hypoxic conditions combined with slow water renewal in the inner bay seemed to play a key role in the polychaete diversity and biomass. Sedimentation processes and organic load coming from untreated sewage into the bay may have negatively affected the survivorship of the fauna.

\section{R E S UM O}

Trinta e oito estações foram amostradas na Baía de Guanabara, Rio de Janeiro, Brasil, no intuito de descrever a diversidade e biomassa de poliquetas sublitorais. As coletas foram realizadas em dois períodos distintos do ano: seco (Setembro 2000) e chuvoso (Maio de 2001). A distribuição espacial dos poliquetas sublitorais demonstrou ser nitidamente heterogênea na Baía de Guanabara. Um gradiente negativo de diversidade e biomassa foi observado em direção as partes internas e protegidas da baía. Foi encontrada uma grande área azóica dentro da baía. Por outro lado, algumas áreas com alta biomassa e baixa diversidade foram encontradas nas proximidades de locais com despejo de esgoto urbano não tratado. Nestas áreas foi observado aumento da biomassa de poliquetas no período chuvoso. Uma comunidade diversificada foi identificada na entrada da baía sem mudanças dramáticas deste padrão entre os dois períodos estudados. Os depositívoros de superfície foram dominantes em toda a área estudada. A importância relativa dos carnívoros e omnívoros aumentou em direção ao setor externo contendo estações de coleta com frações granulométricas mais grosseiras. A Baía de Guanabara pôde ser dividida em três principais regiões levando-se em consideração as condições ambientais, diversidade e biomassa de poliquetas sublitorais: A) área com alta diversidade situada em regiões hidrodinamicamente mais expostas, compostas por areias oxidadas ou pouco reduzidas e com concentrações normais de oxigênio dissolvido na coluna d'água; B) área com baixa diversidade e alta biomassa de poliquetas depositívoras e suspensívoras na porção intermediária da baía próxima a efluentes urbanos, abrangendo estações com contribuições semelhantes de silte, argila e areias finas; C) área azóica ou com emprobecimento da comunidade de poliquetas, apresentando baixa densidade em regiões com baixa energia hidrodinâmica compostas por silte e argila em condição extremamente reduzida, altas concentrações de matéria orgânica total e pouca disponibilidade de oxigênio na coluna d'água, localizada essencialmente do meio até o setor norte da baía. O alto conteúdo de matéria orgânica e as condições de hipoxia da coluna d’água, combinados com a baixa renovação das águas nas áreas protegidas, parecem ter exercido papel fundamental na diversidade e biomassa das poliquetas sublitorais. Os processos de sedimentação e o aporte orgânico intenso a que a Baía de Guanabara está sujeita podem ter contribuído negativamente para a sobrevivência da fauna.

Descriptors: Soft-bottom, Polychaeta, Macroinfauna, Hypoxia, Monitoring programs, Pollution.

Descritores: Substrato inconsolidado, Polychaeta, Macroinfauna, Hipoxia, Programas de monitoramento, Poluição. 


\section{INTRODUCTION}

The description of distribution and abundance patterns of organisms is an essential goal in ecology. Observed patterns are the basis on which models are built, and hypotheses are formed and tested by experiments (MARTIN et al., 1993). Analysis of macrobenthic infauna is also essential in marine environmental monitoring programs (LU et al., 2002). Such analyses of macrobenthos are supported by the considerable number of species collected per sample, the variety of feeding and reproductive habits, and the narrow range of movement, making these animals easily exposed to both contaminants and other disturbances (GRAY, 2002). Thus, the study of the macrobenthos has received considerable attention because of their significance as biological indicators of environmental changes in aquatic systems (DATTA; SARANGI, 1987).

The soft-bottom polychaete fauna may represent up to $70 \%$ of the total abundance and biomass in an ecosystem (GRAY, 1974), and polychaetes are extensively used as a key taxon in bioenvironmental studies to assess natural and humaninduced changes (POCKLINGTON; WELLS, 1992; JONES; KALY, 1996; MUNIZ; PIRES, 2000; GRAY et al., 2002; FARACO; LANA, 2003; VENTURINI et al., 2004).

Until recently, studies of macrozoobenthos conducted in Guanabara Bay have focused mainly on biotopes along its shorelines, such as beaches, mangrove forests and islands (OLIVEIRA, 1958; OLIVEIRA; KRAU, 1976; ANDRADE; MACIEL, 1979; SILVA et al., 1980; VERGARA FILHO et al., 1997). Only one study has examined the spatial distribution and function of sublittoral macroinfauna throughout the bay (FLORES JR. et al., 1979). Therefore, there is a gap in knowledge of the key taxa inhabiting soft bottoms of Guanabara Bay.

With increasing human population growth and human-induced alterations, accurate information on benthic communities is urgently needed for proper management and conservation along coastlines in tropical countries (ALONGI, 1989). The aim of the present study was to assess the sublittoral polychaete macroinfauna diversity and biomass in a grossly polluted urban bay, describing its composition and identifying areas subject to different levels of environmental stress. Our hypothesis was that diversity and biomass are affected by hydrodynamic energy and sediment heterogeneity throughout the bay. We assumed that the polychaete fauna is negatively affected by high amounts of organic matter and pollutants from untreated sewage discharges in the inner parts of the bay, compared with less-polluted areas in the outer parts of the bay.

\section{Material and Methods}

Study Area

Guanabara Bay is located in the second most densely populated state of Brazil, Rio de Janeiro. Its drainage basin extends between $22^{\circ} 24^{\prime} \mathrm{S}$ and $22^{\circ} 57^{\prime} \mathrm{S}$; and $42^{\circ} 33^{\top} \mathrm{W}$ and $43^{\circ} 19^{\prime} \mathrm{W}$. There are two welldefined seasons, a rainy (December to April) and a dry (June to October) period. The bay has a complex bathymetric profile, varying from less than 3 meters in the inner areas to around 58 meters in the main central channel (KJERFVE et al., 1997). Annual mean water temperatures range on average from $25^{\circ} \mathrm{C}$ at the surface to $23.7^{\circ} \mathrm{C}$ in the bottom layer (PARANHOS; MAYR, 1993). Salinity decreases from the outer bay (34.59) towards the inner areas (26.1) (KJERFVE et al., 1997). Paranhos and Mayr (1993) described regional seasonal patterns with lower salinities in the summer and higher salinities in the winter. Sporadically, there is a remarkable change in the pattern described above as a consequence of the penetration of the South Atlantic Central Water (SACW), which fills the bottom of the bay with cold, high-salinity waters $\left(\sim 18^{\circ} \mathrm{C}\right.$ and 36$)$ (KJERFVE et al., 1997).

In general, the bay has calm waters with low swells and gentle winds, predominantly from the east (AMADOR, 1997). This pattern changes when cold fronts enter from the southwest. At these times, swells can reach from 2 to 4 meters with periods from 8 to 12 seconds, resulting in waves that break on the oceanic beaches around the bay mouth.

Sandy sediments cover most of the oceanic part of the bay and are widely distributed towards the natural dredge channels (AMADOR, 1997). Sandy bottoms also occur at some sites near several rivers and from the northwest to southwest parts of Governador Island. A large deposit of mud covers the inner parts, in consequence of the active transport of fluvial clastic materials associated with areas subject to less hydrodynamic energy (KJERFVE et al., 1997).

Guanabara Bay is one of the most impacted ecosystems and the most degraded coastal bay in Brazil (MAYR et al., 1989; PARANHOS et al., 1995). Its basin is densely urbanized, and pollution by untreated domestic sewage is considered to be the worst environmental problem in the bay (MAYR et al., 1989; PARANHOS et al., 1995). Concentrations of coprostanol, sometimes higher than $40 \mu \mathrm{g} \mathrm{g}^{-1}$, indicate areas of severe sewage contamination (CARREIRA et al., 2002). Nowadays, the mean total and fecal coliform values range from $10^{3}$ to $10^{8} \mathrm{~L}^{-1}$ (PARANHOS et al., 1995), while bacterial activity reaches up to $7.35 \mu \mathrm{g} \mathrm{C} \mathrm{L}^{-1} \mathrm{~h}^{-1}$ in the inner part of the 
bay where tidal circulation is restricted (ANDRADE et al., 2003). Guanabara Bay is considered one of the most productive marine ecosystems in the world, with carbon assimilation values varying between 800 and $3600 \mathrm{mg} \mathrm{C}$ day $^{-1}$ and a mean net primary production (NPP) of $0.17 \mathrm{~mol} \mathrm{C} \mathrm{m}^{-2} \mathrm{day}^{-1}$ (REBELLO et al., 1988).

Sampling Strategy and Data Analysis

The present study was carried out in Guanabara Bay, as part of the major project "BIOPLAT - Biodiversity and Biomass of the Brazilian Continental Shelf" (VAN DER VEM et al., 2006; MENDES et al., 2007; SILVA et al., 2008). Two oceanographic surveys, in September 2000 (dry season) and May 2001 (rainy season), were undertaken on board $R / V$ Ursula, sampling 38 stations separated by two nautical miles (Fig. 1). Sampling station positioning was done on board with the help of a Global Positioning System (GPS) model GP - 1800 integrated with a DGPS correction model GR -80 . For the bathymetric data, a high-resolution ecobathymeter model FCV - 582 was used. Surface- and bottom-water variables including temperature, $\mathrm{pH}$, salinity, and dissolved-oxygen concentration were measured at all stations. For this procedure a van Dorn bottle was utilized; for water transparency a Secchi disc was used. Bottom-water samples were taken one meter above the bottom. Salinity was measured by the Strickland \& Parsons method (1968), and dissolved oxygen was obtained by the modified Winkler chemical method (GRASSHOFF et al., 1983) and also the oxygen saturation levels were calculated. Three van Veen grab samples $\left(0.1 \mathrm{~m}^{2}\right)$ were collected at each station to obtain samples for environmental and biological data. Small sediment subsamples (100 g) were collected from the first van Veen grab replicate for sedimentological studies, and an additional 50 grams for determination of total organic matter. All samples were frozen immediately after collection. The redox potential measurements were taken in situ with a simple platinum electrode, A05/AG Analyser ${ }^{\circledR}$ model 6 . The biological sediment samples were sieved on board through a $1.0 \mathrm{~mm}$ mesh, and then fixed with $4 \%$ buffered formalin-seawater.

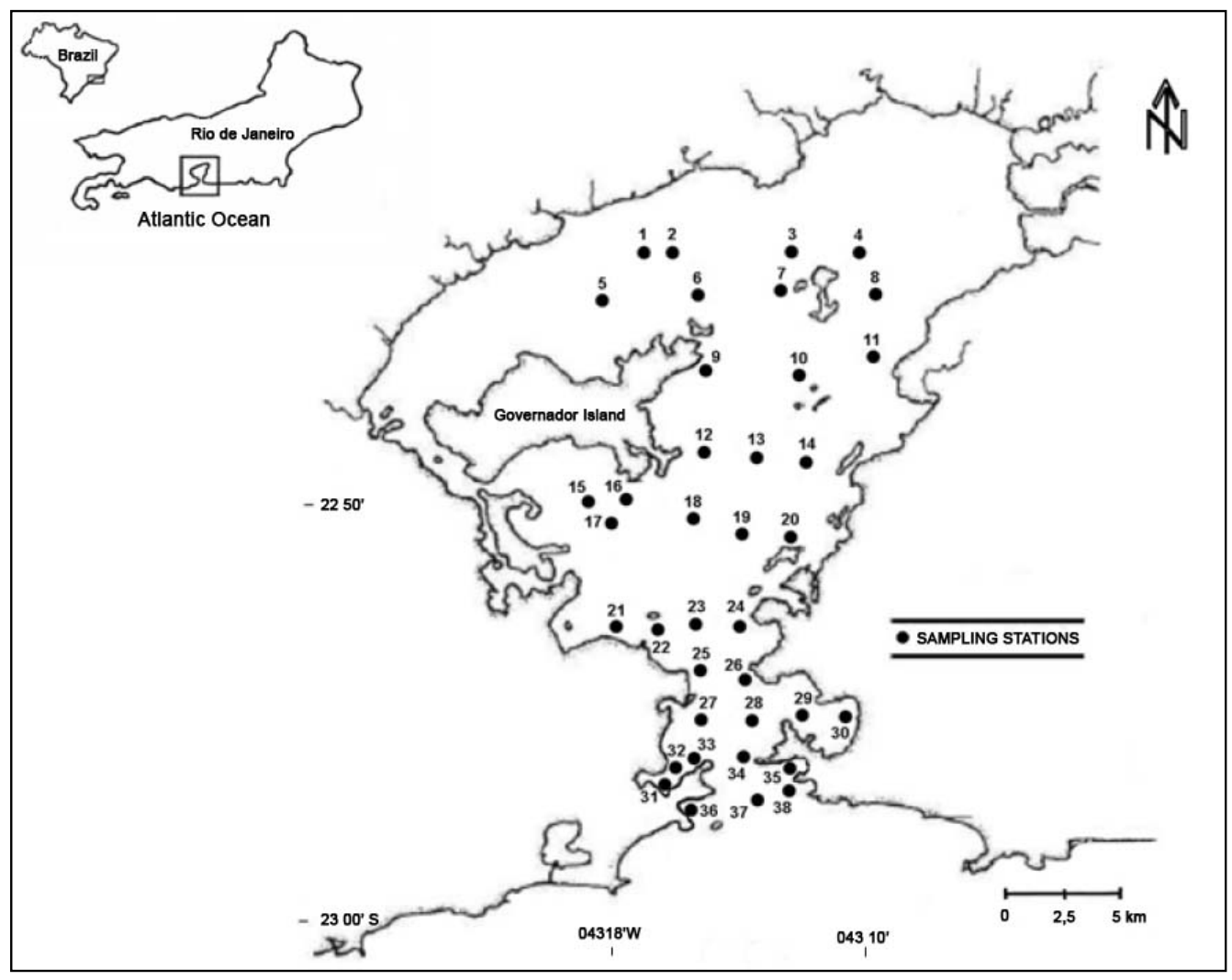

Fig. 1. Map of Guanabara Bay, showing the 38 sampling stations. 
Sediment grain size analysis and carbonate content followed the methodology proposed by Suguio (1973). Total organic matter (TOM) content of the dry sediment was estimated as the loss of weight after ashing (WIDBOM, 1984). The polychaetes were separated, identified to the lowest practicable taxonomic level and then counted by the use of a stereomicroscope (Wild Heerbrug M-8). Total polychaete biomass per station was obtained by determination of the ash-free dry weight (AFDW) expressed in grams per square meter $\left(\mathrm{g} / \mathrm{m}^{2}\right)$. In order to determine the ash-free dry weight, crucibles with previously dried samples were heated to $550^{\circ} \mathrm{C}$ for four hours and weighed three times after cooling in a desiccator. The relative frequency followed Guille (1970). Species richness, Shannon-diversity $\left(\log _{2}\right)$ and evenness were assessed using the PRIMER ${ }^{\circledR} 5$ package (version 5.2.4.; 2001). After a D'Agostino test verified the normality of the data, a Pearson linear correlation index was performed to check the correlation level of the species richness, diversity, evenness and biomass among the analyzed environmental variables with the help of STATISTICA $^{\circledR}$ (Statsoft, INC - version 1999). The modified trophic importance index (Ti) (MUNIZ; PIRES, 1999) was also calculated for five selected trophic categories (deposit feeder, subsurface deposit feeder, suspension feeder, carnivore and omnivore) according to Fauchald and Jumars (1979). After calculation of the $\mathrm{Ti}$ index for each station, the results were then summed and presented as the total trophic group contribution in the three different sectors defined for the bay (inner sector: stations 1 to 11 ; intermediary sector: stations 12 to 26 , and outer sector: stations 27 to 38 ).

Principal Components Analysis (PCA) was carried out for centered environmental data from both sampling periods, using the FITOPAC $^{\odot}$ program (Multi-Variate Statistical Package) (George Shepard State University of Campinas, 1995). Polychaete sampling station affinities were assessed by a BrayCurtis similarity routine, established by the use of the average group link of unweighted means after a logtransformation $(\log x+1)$ of the polychaete abundance data. Those stations that contained less than $10 \%$ of the highest polychaete density were considered as impoverished or azoic areas, and were excluded from the analyses to avoid misinterpretation of the results of the sampling-station affinities. To test the hypothesis that the groups of sampling stations formed in the cluster analysis were different, a one-way analysis of similarity (ANOSIM) was carried out using the BrayCurtis similarity matrix.

The SIMPER test (Similarity Percentages species contributions) was performed for both dry and rainy seasons, following the Bray-Curtis polychaete abundance groups identified in the ANOSIM test. The
BIOENV test (Biota-Environmental Matching) was applied to assess affinity between the species abundance and environmental matrix data for both seasons by the Spearman rank correlation method (CLARKE; WARWICK, 1994). Environmental data matrices were standardized and linked by average group link by unweighted means for a similarity matrix separated by Euclidean distance.

\section{RESULTS}

Environmental Features

The main environmental variables that drove the groupings of sampling stations were almost the same between the two periods studied. In both dry and rainy seasons, silt and clay fractions, dissolved oxygen saturation and sand fractions were responsible for the sampling station groups formed. As shown in the PCA analysis (Fig. 2), factorial axes 1 and 2 were responsible for $85 \%$ of the variance in the dry season and $84 \%$ in the rainy season. At most of the sampling stations (> 52\%), oxygen content was low in the bottom layer, but highly saturated at the surface (Table 1). Sediment features, carbonates and total organic matter indicated that Guanabara Bay has complex habitat heterogeneity for the polychaete fauna (Table 2).

Group A combined those sites that shared essentially the normoxic water-column conditions, and sediments composed of coarse and medium sands with some contribution of biogenic carbonates. This group of stations was concentrated around the bay mouth. Group B also showed high oxygen contents, but grouped deeper stations with fine to very fine, poorly sorted sands. These sampling stations are located around the central channel in the middle parts of the bay. Group $\mathrm{C}$ grouped stations located at sites with a low-energy hydrodynamic regime and large amounts of total organic matter. In both periods, silt and clay fractions accounted for these sampling-station groupings. The granulometric conditions of Group C revealed poorly sorted sediments with a major contribution of silt and clay fractions, large amounts of total organic matter (mean TOM $=14 \%$ ), with an extremely reduced sediment (mean $\mathrm{Eh}=-350 \mathrm{mV}$ ). Hypoxic conditions (mean $1.40 \mathrm{~mL} / \mathrm{L}$ ) of the bottom water were recorded at all group $\mathrm{C}$ stations.

\section{Polychaete Community Composition}

A total of 9,021 individuals belonging to 77 polychaete species and 34 families were identified (Table 3). Less than 5\% of all collected species accounted for over $80 \%$ of the total abundance. Only five families (Spionidae, Onuphidae, Goniadidae, Capitellidae and Sabellidae) had more than two 
species per family, many of them sporadic and rare. Rare species were mainly found in the outer parts of the bay, in the bay mouth, and in the middle sector around the central channel. In the rainy season, polychaete abundance was higher (5,523 ind.) than in the dry season $(3,498$ ind.) No great changes were observed in the polychaete species collected between one season and another.
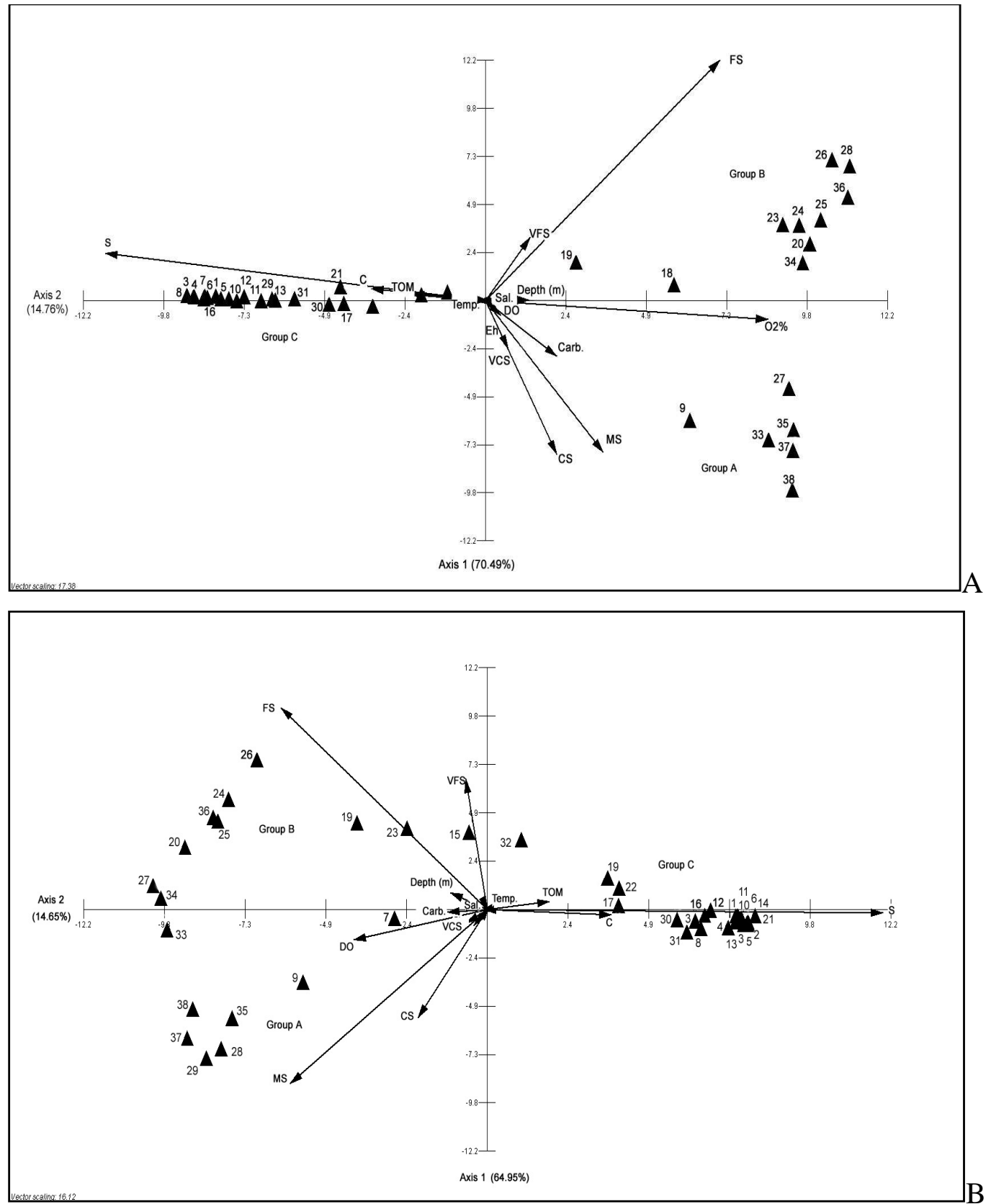

Fig. 2. Biplot diagram of the Principal Component Analysis (PCA) of environmental variables analyzed and the groups A, B and $\mathrm{C}$ formed in the dry (A) and rainy (B) seasons. Small vectors are omitted. Numbers 1 to 38 refer to sampling stations. $02 \%$, percent dissolved oxygen; Carb., carbonates, CS, coarse sand, FS, fine sand; MS, medium sand; VFS, very fine sand; TOM, total organic matter. Percentage of explained variance by the first two axes is also indicated in the figure. 
Table 1. Environmental water column variables studied in the 38 sampling stations of the Guanabara Bay, in dry and rainy season. Depth, Water transparency (Secchi disc depth), dissolved oxygen $(\mathrm{O} 2(\mathrm{~mL} / \mathrm{L})$, percent oxygen saturation $(\mathrm{O} 2 \%)$.

\begin{tabular}{|c|c|c|c|c|c|c|c|}
\hline \multirow[b]{3}{*}{ Stations } & \multirow[b]{3}{*}{ Depth } & \multirow{2}{*}{\multicolumn{2}{|c|}{$\frac{\text { Water transparency }}{\text { (meters) }}$}} & \multirow{2}{*}{\multicolumn{2}{|c|}{$\frac{\mathrm{O}_{2}(\mathrm{~mL} / \mathrm{L})}{\mathrm{Dry}}$}} & \multirow{2}{*}{\multicolumn{2}{|c|}{$\frac{\mathrm{O}_{2} \% \text { saturation }}{\text { Rainy }}$}} \\
\hline & & & & & & & \\
\hline & & Dry & Rainy & Surface & Bottom & Surface & Bottom \\
\hline 1 & 3,15 & 0,70 & 1,00 & 8,36 & 1,11 & 129 & 53 \\
\hline 2 & 3,90 & 0,75 & 0,90 & 7,75 & 0,81 & 96 & 60 \\
\hline & 5,25 & 0,65 & 1,05 & 10,90 & 0,81 & 97 & 69 \\
\hline 4 & 5,40 & 0,80 & 0,80 & 11,92 & 0,94 & 151 & 51 \\
\hline 5 & 3,40 & 0,60 & 0,65 & 6,45 & 1,66 & 71 & 55 \\
\hline 6 & 7,25 & 1,05 & 1,05 & 6,65 & 1,19 & 79 & 59 \\
\hline 7 & 5,90 & 0,90 & 1,20 & 10,19 & 1,14 & 96 & 75 \\
\hline 8 & 5,10 & 0,65 & 0,70 & 10,24 & 1,04 & 152 & 72 \\
\hline 9 & 7,50 & 0,80 & 1,75 & 7,88 & 3,39 & 61 & 57 \\
\hline 10 & 8,30 & 0,50 & 1,60 & 13,47 & 1,01 & 106 & 50 \\
\hline 11 & 3,50 & 0,60 & 0,60 & 5,79 & 2,32 & 191 & 68 \\
\hline 12 & $\begin{array}{l}3,90 \\
4,90\end{array}$ & $\begin{array}{l}0.00 \\
1,75\end{array}$ & $\begin{array}{l}1,40 \\
1,45\end{array}$ & 5,40 & $\begin{array}{l}\text {, } 1,56 \\
1,56\end{array}$ & 44 & $\begin{array}{l}\text { lo } \\
33\end{array}$ \\
\hline 13 & $\begin{array}{l}\begin{array}{l}6,90 \\
6,60\end{array}\end{array}$ & $\begin{array}{l}1,75 \\
1,85\end{array}$ & $\begin{array}{l}1,45 \\
1,00\end{array}$ & $\begin{array}{l}5,40 \\
5,67\end{array}$ & $\begin{array}{l}1,36 \\
2,40\end{array}$ & $\begin{array}{l}44 \\
58\end{array}$ & $\begin{array}{l}33 \\
46\end{array}$ \\
\hline 14 & $\begin{array}{c}\text {, } \\
11,50\end{array}$ & $\begin{array}{l}1,80 \\
1,25\end{array}$ & $\begin{array}{l}1,00 \\
0,00\end{array}$ & $\begin{array}{l}\text {,6/ } \\
5,72\end{array}$ & $\begin{array}{l}2,40 \\
3,70\end{array}$ & $\begin{array}{l}58 \\
55\end{array}$ & $\begin{array}{l}46 \\
50\end{array}$ \\
\hline $\begin{array}{l}14 \\
15\end{array}$ & $\begin{array}{l}11,50 \\
11,40\end{array}$ & $\begin{array}{l}1,25 \\
0,00\end{array}$ & 0 & $\begin{array}{l}5,72 \\
10,32\end{array}$ & $\begin{array}{l}3,0 \\
3,08\end{array}$ & $\begin{array}{l}55 \\
85\end{array}$ & $\begin{array}{l}50 \\
54\end{array}$ \\
\hline 16 & 6,80 & 0,85 & 1,60 & 7,85 & 1,56 & 80 & \\
\hline 17 & 5,80 & 220 & 1,00 & $\begin{array}{l}1,05 \\
4,64\end{array}$ & $\begin{array}{l}1,90 \\
1,90\end{array}$ & 50 & $\begin{array}{l}04 \\
38 \\
38\end{array}$ \\
\hline 18 & 17,10 & $\begin{array}{l}1,50 \\
1,50\end{array}$ & 2,85 & 6,29 & 4,44 & 73 & 60 \\
\hline 10 & 131, & 100 & 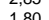 & 5 & 4,44 & 85 & 63 \\
\hline 20 & 3,10 & 1,05 & 1,55 & 6,52 & 4,03 & 98 & 97 \\
\hline 21 & $\begin{array}{l}0,10 \\
4,40\end{array}$ & 105 & 250 & 3.57 & $\begin{array}{l}4,00 \\
234\end{array}$ & 74 & 52 \\
\hline 27 & 933 & 190 & 230 & 400 & 395 & 85 & 68 \\
\hline 22 & , & , 1,90 & ג, 200 & $\begin{array}{l}4,00 \\
\end{array}$ & ,, 93 & $\begin{array}{l}63 \\
709\end{array}$ & 60 \\
\hline 24 & $\begin{array}{l}28,30 \\
3,50\end{array}$ & $\begin{array}{l}1,18 \\
1,90\end{array}$ & $\begin{array}{l}2,20 \\
2,50\end{array}$ & $\begin{array}{l}4,92 \\
5,56\end{array}$ & $\begin{array}{l}4,09 \\
4,50\end{array}$ & 91 & 71 \\
\hline 25 & $\begin{array}{r}23,70 \\
23,70\end{array}$ & 2,00 & 2,55 & 4.17 & $\begin{array}{l}4,30 \\
4,31\end{array}$ & 95 & 88 \\
\hline 26 & 9,30 & 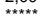 & 2,90 & 3,95 & 3,86 & 71 & 71 \\
\hline 27 & 10,50 & 2,25 & 1,80 & 4,39 & 4,12 & 91 & 91 \\
\hline 28 & 8,10 & 4,10 & 4,10 & 3,86 & 3,81 & 69 & 67 \\
\hline 29 & 5,40 & 3,30 & 2,00 & 3,92 & 2,30 & 91 & 90 \\
\hline 30 & 2,75 & 2,00 & 0,90 & 4,59 & 3,67 & 78 & 69 \\
\hline 31 & 8,20 & 4,35 & 1,95 & 3,64 & 2,69 & 105 & 93 \\
\hline $\begin{array}{l}11 \\
32 \\
\end{array}$ & $\begin{array}{l}\text {, } 20,50 \\
10,50\end{array}$ & $\begin{array}{l}\text {, } 4,55 \\
4,55\end{array}$ & 0 & $\begin{array}{l}3,04 \\
3,86\end{array}$ & $\begin{array}{l}3,09 \\
3,67\end{array}$ & 88 & 45 \\
\hline $\begin{array}{l}32 \\
33 \\
\end{array}$ & 9,20 & $\begin{array}{l}4,55 \\
3,15\end{array}$ & 2,80 & $\begin{array}{l}3,86 \\
3,86\end{array}$ & $\begin{array}{l}3,61 \\
3,86\end{array}$ & $\begin{array}{l}88 \\
83\end{array}$ & $\begin{array}{l}45 \\
82\end{array}$ \\
\hline 34 & $\begin{array}{l}3,<0 \\
31,40\end{array}$ & 2,05 & 2,00 & 5 & 4,61 & 90 & 75 \\
\hline $\begin{array}{l}34 \\
35\end{array}$ & $\begin{array}{l}31,40 \\
12,50\end{array}$ & $\begin{array}{l}2,05 \\
3,05\end{array}$ & $\begin{array}{l}2,00 \\
3,00\end{array}$ & $\begin{array}{l}5,85 \\
5,56\end{array}$ & $\begin{array}{l}4,61 \\
4,52\end{array}$ & 91 & $\begin{array}{l}15 \\
82\end{array}$ \\
\hline 36 & 9,30 & 5,55 & 4,50 & 5,02 & 4,56 & 98 & 87 \\
\hline 37 & 12,10 & $\begin{array}{l}1,65 \\
1,65\end{array}$ & 1,70 & 6,70 & 4,73 & 99 & 89 \\
\hline 38 & 18.50 & $\begin{array}{l}2,15 \\
\end{array}$ & $\begin{array}{l}3.00 \\
3.00\end{array}$ & 5,99 & $\begin{array}{l}4,56 \\
\end{array}$ & 101 & 88 \\
\hline
\end{tabular}

Table 2. Granulometry, carbonates and total organic matter observed in both studied periods. Note: Carb., carbonates, CS, coarse sand, FS, fine sand; MS, medium sand; VFS, very fine sand; TOM, total organic matter.

\begin{tabular}{|c|c|c|c|c|c|c|c|c|c|c|c|c|c|c|c|c|c|c|}
\hline \multirow[b]{2}{*}{ Station } & \multicolumn{2}{|c|}{ VCS (\%) } & \multicolumn{2}{|c|}{ CS $(\%)$} & \multicolumn{2}{|c|}{ MS (\%) } & \multicolumn{2}{|c|}{ FS (\%) } & \multicolumn{2}{|c|}{ VFS $(\%)$} & \multicolumn{2}{|c|}{ Silt (\%) } & \multicolumn{2}{|c|}{ Clay $(\%)$} & \multicolumn{2}{|c|}{ Carb.(\%) } & \multicolumn{2}{|c|}{ TOM (\%) } \\
\hline & Dry & Rainy & Dry & Rainy & Dry & Rainy & Dry & Rainy & Dry & Rainy & Dry & Rainy & Dry & Rainy & Dry & Rainy & Dry & Rainy \\
\hline 1 & 0.00 & 0.00 & 0.00 & 0.00 & 0.00 & 0.00 & 0.00 & 0.00 & 1.86 & 1.51 & 67.38 & 71.62 & 30.76 & 26.87 & 0 & 0.00 & $\frac{16.94}{16.94}$ & 16.83 \\
\hline 2 & 0.00 & 0.00 & 0.00 & 0.00 & 0.00 & 0.00 & 0.00 & 0.00 & 0.24 & 0.99 & 66.55 & 79.56 & 33.21 & 19.44 & 0 & 0.00 & 18.62 & 15.84 \\
\hline 3 & 0.00 & 0.00 & 0.00 & 0.00 & 0.00 & 0.00 & 0.00 & 0.00 & 1.23 & 5.86 & 72.09 & 68.46 & 26.68 & 25.67 & 0 & 0.00 & 16.8 & 14.03 \\
\hline 4 & 0.00 & 0.00 & 0.00 & 0.00 & 0.00 & 0.00 & 0.00 & 0.00 & 0.17 & 1.12 & 74.42 & 75.80 & 25.40 & 23.08 & 0 & 0.00 & 15.29 & 14.31 \\
\hline 5 & 0.00 & 0.00 & 0.00 & 0.00 & 0.00 & 0.00 & 0.00 & 0.00 & 0.07 & 0.90 & 76.68 & 77.74 & 20.26 & 21.35 & 0 & 0.00 & 13.32 & 12.92 \\
\hline 6 & 0.00 & 0.00 & 0.00 & 0.00 & 0.00 & 0.00 & 0.00 & 0.00 & 0.80 & 0.77 & 74.50 & 79.20 & 24.70 & 20.03 & 0 & 0.00 & 16 & 9.98 \\
\hline 7 & 0.00 & 12.62 & 0.00 & 7.57 & 0.00 & 13.88 & 0.00 & 17.67 & 2.47 & 8.83 & 76.24 & 29.59 & 21.29 & 8.58 & 0 & 81.80 & 16.72 & 2.60 \\
\hline 8 & 0.00 & 0.00 & 0.00 & 0.00 & 0.00 & 0.00 & 0.00 & 0.00 & 0.43 & 1.14 & 79.19 & 69.35 & 20.38 & 29.51 & 0 & 0.00 & 17.12 & 12.96 \\
\hline 9 & 38.90 & 24.88 & 29.74 & 29.94 & 17.12 & 26.76 & 6.01 & 11.57 & 0.75 & 1.01 & 2.59 & 2.49 & 2.03 & 1.62 & 13.5 & 6.30 & 1.5 & 1.56 \\
\hline 10 & 0.00 & 0.00 & 0.00 & 0.00 & 0.00 & 0.00 & 0.00 & 0.00 & 2.44 & 4.89 & 60.41 & 74.49 & 37.15 & 20.63 & 0.3 & 1.00 & 14.9 & 12.94 \\
\hline 11 & 0.00 & 0.00 & 0.00 & 0.00 & 0.00 & 0.00 & 0.00 & 0.00 & 0.36 & 0.78 & 78.65 & 76.84 & 20.99 & 22.38 & 0.2 & 0.00 & 13.88 & 11.82 \\
\hline 12 & 0.00 & 0.00 & 0.00 & 0.00 & 0.00 & 0.00 & 0.00 & 0.00 & 7.38 & 5.81 & 73.05 & 56.45 & 19.57 & 37.75 & 1.5 & 1.10 & 14.09 & 11.41 \\
\hline 13 & 0.00 & 0.00 & 0.00 & 0.00 & 0.00 & 0.00 & 0.00 & 0.00 & 3.50 & 4.17 & 75.06 & 71.02 & 21.44 & 24.81 & 0.3 & 0.00 & 13.31 & 13.00 \\
\hline 14 & 0.00 & 0.00 & 0.00 & 0.00 & 0.00 & 0.00 & 0.00 & 0.00 & 7.45 & 2.75 & 66.35 & 77.95 & 26.20 & 19.30 & 0.1 & 4.20 & 14.92 & 4.67 \\
\hline 15 & 0.00 & 1.17 & 0.00 & 0.00 & 6.02 & 3.50 & 12.04 & 26.87 & 2.58 & 29.79 & 54.09 & 32.93 & 25.27 & 5.73 & 1.6 & 2.80 & 12.67 & 4.56 \\
\hline 16 & 0.00 & 0.00 & 0.00 & 0.00 & 0.00 & 0.00 & 0.00 & 0.00 & 1.94 & 8.34 & 81.15 & 71.17 & 16.91 & 20.49 & 0.2 & 0.00 & 11.81 & 12.10 \\
\hline 17 & 3.77 & 4.03 & 3.14 & 4.03 & 3.77 & 5.38 & 6.28 & 8.06 & 3.77 & 8.06 & 62.85 & 50.72 & 16.41 & 17.02 & 3.7 & 7.20 & 7.78 & 10.82 \\
\hline 18 & 3.13 & 2.46 & 5.21 & 2.46 & 11.20 & 8.00 & 27.61 & 29.23 & 21.10 & 41.84 & 24.94 & 13.51 & 6.30 & 2.20 & 1.7 & 2.30 & 6.36 & 4.51 \\
\hline 19 & 0.25 & 0.00 & 0.50 & 0.00 & 1.76 & 0.00 & 22.84 & 5.40 & 26.10 & 26.12 & 42.16 & 53.51 & 6.39 & 13.17 & 1.7 & 0.80 & 5.56 & 11.01 \\
\hline 20 & 1.08 & 0.37 & 2.63 & 1.67 & 21.87 & 24.25 & 56.29 & 52.57 & 14.34 & 19.07 & 1.78 & 1.58 & 1.90 & 0.50 & 1.9 & 1.10 & 1.3 & 1.96 \\
\hline 21 & 0.00 & 0.00 & 0.00 & 0.00 & 2.18 & 0.00 & 9.81 & 0.00 & 3.27 & 5.33 & 62.87 & 80.66 & 21.87 & 14.01 & 1 & 0.00 & 11.31 & 15.73 \\
\hline 22 & 0.00 & 0.00 & 0.00 & 0.00 & 3.34 & 0.91 & 11.34 & 5.43 & 4.67 & 21.73 & 58.45 & 57.98 & 22.21 & 13.95 & 0.7 & 0.00 & 13.33 & 10.83 \\
\hline 23 & 0.59 & 0.60 & 1.56 & 0.00 & 5.48 & 5.42 & 52.03 & $\begin{array}{l}34.32 \\
\end{array}$ & 20.34 & 25.29 & 12.51 & 27.61 & 6.90 & 6.77 & 8.8 & 0.60 & 3.41 & 8.88 \\
\hline 24 & 2.84 & 0.68 & 3.02 & 2.48 & 10.29 & 12.16 & 58.37 & 64.64 & 11.53 & 14.19 & 7.18 & 3.89 & 5.17 & 1.52 & 3.9 & 3.00 & 3.07 & 1.97 \\
\hline 25 & 0.95 & 2.07 & 2.54 & 3.11 & 12.68 & 13.74 & 63.73 & 59.62 & 8.72 & 9.85 & 6.59 & 6.97 & 4.63 & 3.60 & 10.3 & 15.40 & 2.44 & 5.03 \\
\hline 26 & 0.14 & 0.22 & 0.14 & 0.22 & 3.06 & 0.65 & 79.98 & 54.70 & 12.66 & 41.51 & 2.01 & 0.50 & 2.01 & 2.21 & 2.2 & 2.50 & 1.42 & 3.31 \\
\hline 27 & 0.36 & 0.00 & 6.14 & 1.02 & 61.96 & 39.66 & 25.50 & 54.74 & 0.24 & 4.07 & 3.09 & 1.04 & 2.59 & 0.52 & 8.6 & 4.60 & 0.92 & 1.40 \\
\hline 28 & 0.39 & 0.84 & 0.66 & 31.07 & 8.79 & 59.61 & 83.27 & 7.92 & 5.64 & 0.24 & 0.31 & 0.12 & 0.94 & 0.08 & 1.2 & 1.40 & 0.25 & 0.76 \\
\hline 29 & 0.00 & 0.90 & 0.00 & 1.61 & 0.00 & 68.99 & 0.00 & 0.00 & 5.89 & 0.09 & 75.51 & 0.12 & 18.60 & 0.16 & 0 & 12.20 & 12.57 & 1.30 \\
\hline 30 & 0.00 & 0.00 & 0.00 & 0.00 & 0.00 & 0.00 & 0.00 & 0.00 & 2.42 & 7.30 & 78.79 & 63.20 & 18.79 & 29.49 & 0.2 & 0.80 & 14.7 & 10.02 \\
\hline 31 & 0.00 & 0.00 & 0.00 & 0.00 & 0.00 & 0.00 & 0.00 & 0.00 & 8.21 & 2.67 & 76.68 & 76.41 & 15.10 & 20.92 & 0.4 & 0.20 & 13.18 & 11.92 \\
\hline 32 & 0.00 & 0.00 & 0.00 & 0.54 & 0.00 & 1.71 & 0.00 & 19.02 & 5.50 & 32.79 & 76.63 & 35.06 & 17.87 & 10.87 & 0.7 & $\begin{array}{l}1.60 \\
1.60\end{array}$ & 15.81 & 10.27 \\
\hline 33 & 8.45 & 0.15 & 25.10 & 3.63 & 40.78 & 49.52 & 11.82 & 44.53 & 2.41 & 0.45 & 0.60 & 1.49 & 4.09 & 0.22 & 49.4 & 5.40 & 1.36 & 1.01 \\
\hline 34 & 0.42 & 0.62 & 2.50 & 5.35 & 12.92 & 41.58 & 52.09 & 49.61 & 9.17 & 2.68 & 18.99 & 0.12 & 3.92 & 0.04 & 50 & 20.50 & 0.55 & 0.78 \\
\hline 35 & 1.77 & 1.86 & 44.18 & 51.79 & 31.50 & 32.28 & 14.59 & 11.15 & 5.32 & 2.55 & 0.47 & 0.12 & 2.03 & 0.14 & 18.8 & 2.70 & 3.46 & 0.40 \\
\hline 36 & 0.00 & 0.14 & 0.15 & 1.40 & 1.69 & 16.35 & 60.01 & 64.26 & 35.85 & 9.22 & 0.61 & 8.63 & 1.68 & 0.01 & 10.8 & 4.90 & 0.36 & 0.70 \\
\hline 37 & $\begin{array}{l}15.72 \\
\end{array}$ & 4.44 & 31.66 & 25.44 & 43.15 & 57.68 & 6.40 & $\begin{array}{l}12.28 \\
\end{array}$ & 0.11 & 0.03 & 0.11 & 0.05 & 1.02 & 0.03 & $\begin{array}{l}3.6 \\
3.6\end{array}$ & 1.70 & 0.27 & 0.58 \\
\hline 38 & 2.36 & 3.47 & 49.91 & 31.11 & 44.72 & 46.30 & 1.18 & 18.66 & 0.12 & 0.24 & 0.35 & 0.00 & 1.12 & 0.10 & 15 & 1.80 & 0.8 & 0.42 \\
\hline
\end{tabular}


Table 3. Species list of the polychaete fauna recorded in the studied area during dry and rainy seasons.

\begin{tabular}{|c|c|}
\hline Mediomastus californiensis Hartman, 1944 & Sigambra grubii Fritz Müller 1858 \\
\hline Notomastus lobatus Hartman, 1947 & Parandalia americana (Hartman, 1947) \\
\hline Capitella capitata (Facricius, 1780) & Pisionidens indica (Aiyar \& Alikunhi, 1940) \\
\hline Euclymene santanderensis Rioja, 1917 & Harmothoe lunulata (Delle Chiaje, 1841) \\
\hline Euclymene oerstedi (Claparède, 1863) & Lepidonotus sp. \\
\hline Ophelia formosa (Kinberg) 1866 & Sthenelanella atypica Berkeley \& Berkeley, 1941 \\
\hline Armandia maculata (Webster) 1884 & Sigalion taquari Amaral \& Nonato, 1984 \\
\hline Scoloplos (Leodamas) johnstonei Day, 1934 & Sthenelais sp. \\
\hline Califia sp. & Eusyllis lamelligera Marion \& Bobretzky, 1875 \\
\hline Naineris setosa (Verril, 1900) & Trypanosyllis parvidentata Perkins, 1981 \\
\hline Scoloplos sp. & Owenia fusiformis Delle Chiaje, 1844 \\
\hline Aricidea suecica simplex Day, 1963 & Chone insularis Nonato, 1981 \\
\hline Aricidea sp. & Megalomma bioculatum (Ehlers, 1887) \\
\hline Protodorvillea biarticulata Day, 1963 & Sabella microphthalma Verril, 1873 \\
\hline Schistomeringos rudolphi (Delle Chiaje, 1828) & Serpula vermicularis Linnaeus, 1767 \\
\hline Protodorvillea sp. & Spiochaetopterus nonatoi Bhaud \& Petti, 2001 \\
\hline Eunice rubra Grube, 1856 & Magelona riojai Jones, 1963 \\
\hline Marphysa sangüinea (Montagu, 1815) & Poecilochaetus australis Nonato, 1963 \\
\hline Lumbrineris tetraura (Schmarda, 1861) & Prionospio heterobranchia Moore, 1907 \\
\hline Ninoe brasiliensis Kinberg, 1865 & Paraprionospio pinnata (Ehlers, 1901) \\
\hline Lumbrineriopsis mucronata (Ehlers, 1908) & Aonides mayaguezensis Foster, 1969 \\
\hline Kinbergonuphis tenuis (Hansen, 1882) & Dispio uncinata Hartman, 1951 \\
\hline Diopatra cuprea (Bosc, 1802) & Prionospio malmgreni Claperède, 1870 \\
\hline Nothria sp. & Spiophanes missionensis Hartman, 1941 \\
\hline Onuphis eremita Audouin and Milne Edwards, 1833 & Spio filicornis (Müller, 1776) \\
\hline Rhamphobrachium sp. & Polydora sp. \\
\hline Pseudoeurythoe ambígua (Monro, 1933) & Spio sp. \\
\hline Glycera americana Leidy, 1855 & Aonides sp. \\
\hline Hemipodus olivieri Orensanz \& Gianluca, 1974 & Isolda puelcha Müller, 1858 \\
\hline Goniadides carolinae Day, 1973 & Cirriformia tentaculata (Montagu, 1808) \\
\hline Goniada maculata Oersted, 1843 & Tharyx sp. \\
\hline Glycinde multidens Fritz Müller, 1858 & Piromis arenosus Kinberg, 1867 \\
\hline Podarke obscura Verril, 1873 & Pectinaria regalis Verril, 1901 \\
\hline Nepthys squamosa Ehlers, 1887 & Thelepus plagiostoma (Schmarda, 1861) \\
\hline Neanthes sp.A & Polycirrus sp. \\
\hline Neanthes sp.B & Loimia medusa (Savigny, 1820) \\
\hline Ceratocephale oculata Banse, 1977 & Terebellides anguicomus Müller, 1858 \\
\hline \multicolumn{2}{|l|}{ Phyllodoce sp. } \\
\hline Eumida sanguinea Oersted, 1843 & \\
\hline Eteone sp. & \\
\hline
\end{tabular}

Figure 3 shows the species richness, abundance, Shannon-diversity (H') and evenness (J'). Species richness, diversity and evenness diminished dramatically towards the more-sheltered areas. Species diversity was high near the bay mouth and around the central channel. The general pattern of these parameters did not change between the two seasons studied. 

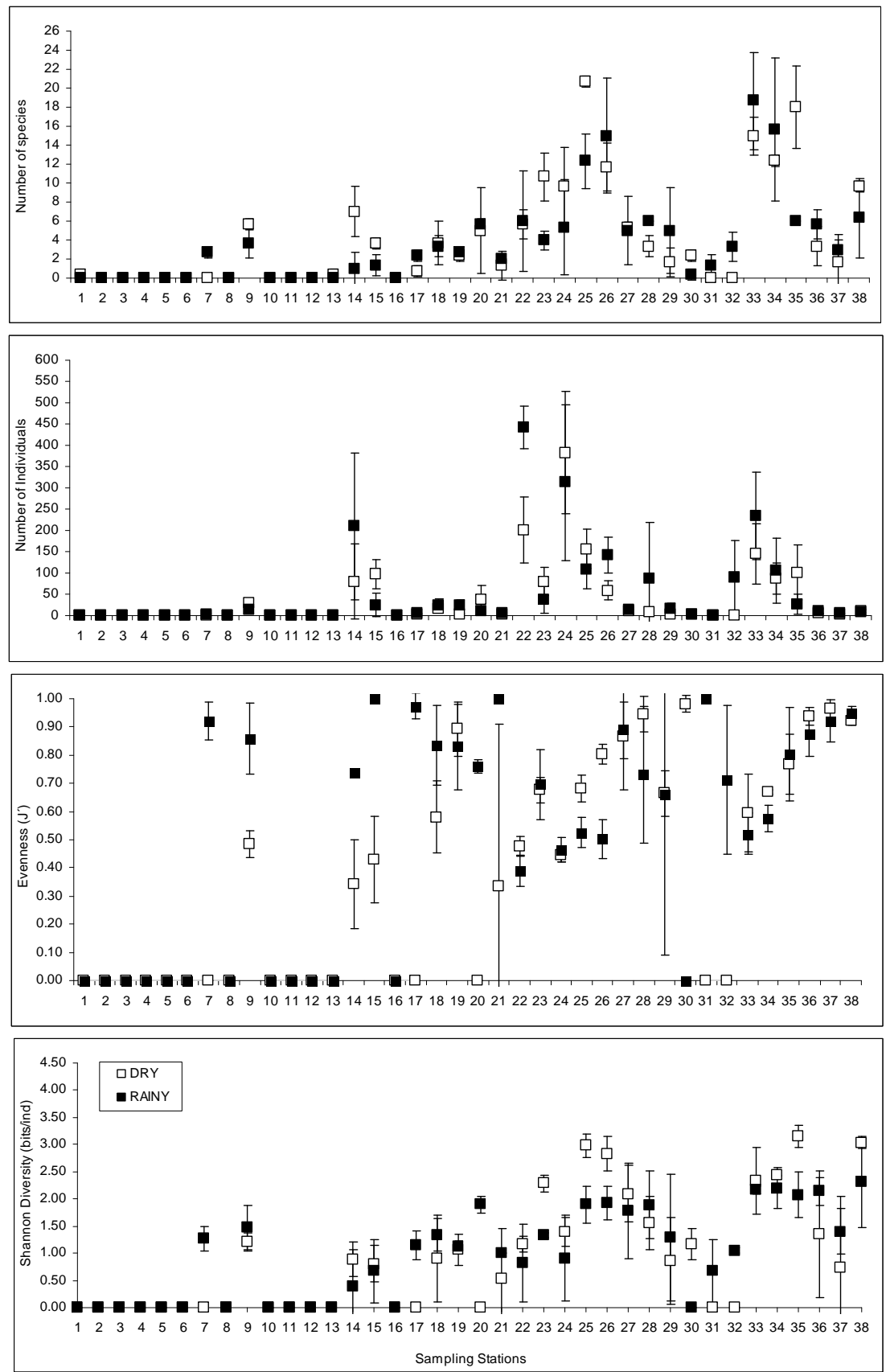

Fig. 3. Polychaete species richness, abundance, evenness (J') and Shannon-diversity (H') recorded in the 38 stations in both study periods at Guanabara Bay. Error bars represent one standard deviation. 
Polychaete abundance was spatially variable throughout the bay. With regard to mean abundance, in the inner part only 69 individuals were found, the middle part yielded 3,150 individuals and the outer part 1,291 . In the rainy season a remarkable change in the abundance of polychaetes was found in the middle part of the bay. Whereas in the dry season, 3,498 individuals were collected, in the rainy season the polychaete abundance was $58 \%$ higher, with 5,523 specimens counted. Poecilochaetus australis Nonato, 1963 and Spiochaetopterus nonatoi Bhaud \& Petti, 2001 were the two most abundant species. Spatially, these two species were found mainly in muddy bottoms of the middle sector.

The highest values of polychaete biomass were found in the middle sector of the bay, with an ash-free dry weight of 18 grams per square meter (Fig. 4). The highest value $\left(41 \mathrm{~g} / \mathrm{m}^{2}\right)$ was recorded in the rainy season at station 22 .

The Pearson linear correlation for the dry season (Table 4) showed a positive correlation of depth, dissolved oxygen, redox potential, sands (except very fine sands), and carbonates with species richness and Shannon-diversity. Negative correlations of species richness and diversity with water temperature, sorting coefficient, silt, clay and TOM were recorded. Polychaete biomass was correlated positively with oxygen, medium and fine sands and carbonates, and negatively with redox potential, temperature, silt and total organic matter (TOM). Except for carbonates, no great changes were recorded in the rainy season (Table 4 ).

The Trophic Index (Ti) revealed that deposit feeders comprised the most important trophic group, followed by carnivores, suspension feeders and omnivores (Table 5). The polychaete trophic structure in the bay was essentially composed by deposit feeders in the inner sectors, suspension feeders, deposit feeders and carnivores in the middle sector, and a more balanced contribution of all the trophic groups in the outer sector. Spionidae and Poecilochaetidae were the two most important polychaete families in the deposit-feeder category. Subsurface deposit-feeding species, represented mainly by the family Maldanidae, showed no important contribution in any part of the bay. In general, the trophic structure did not show a strong change between both study periods, in spite of the slightly increased contribution of deposit feeders in the rainy season in the middle and outer sectors. Omnivores, represented mainly by onuphids, were slightly better represented in the dry season.

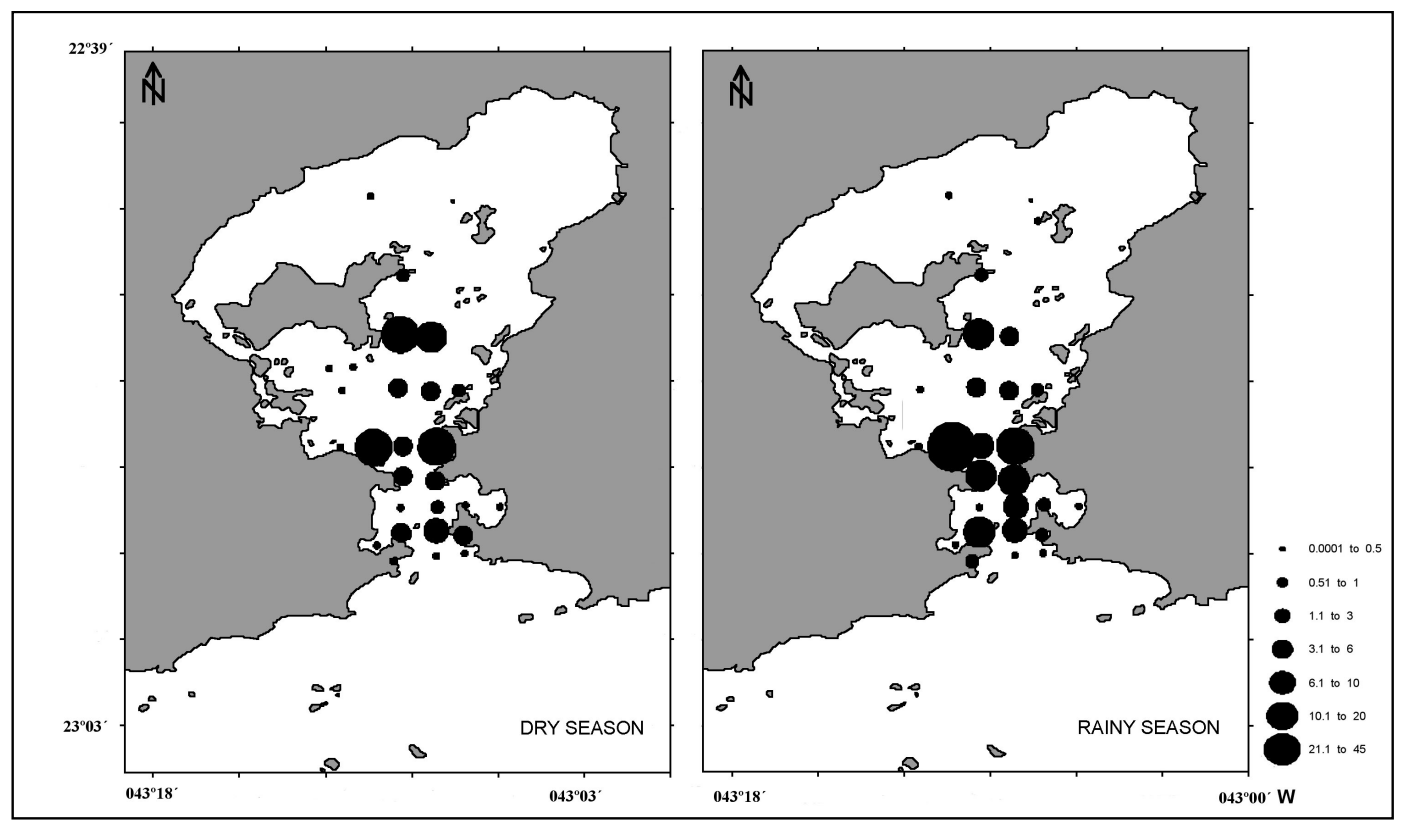

Fig. 4. Polychaete biomass (Ash-free dry weight in grams per meter square) recorded in the study area on dry and rainy periods. Note: stations with zero biomass values were omitted from the map. 
Table 4. Pearson linear correlation between Shannon-diversity (H'), Pielou's evenness (J), species richness (R), biomass (B) and environmental variables analyzed in the dry and rainy seasons. Bold indicates significant values $(\mathrm{p}<0.005)$.

\begin{tabular}{lcccccccc}
\hline & \multicolumn{2}{c}{$\mathrm{R}$} & \multicolumn{2}{c}{$\mathrm{H}^{\prime}$} & \multicolumn{2}{c}{$\mathrm{J}$} & \multicolumn{2}{c}{$\mathrm{B}(\mathrm{g} / \mathrm{m} 2)$} \\
\hline Environmental variables & Dry & Rainy & Dry & Rainy & Dry & Rainy & Dry & Rainy \\
\hline Depth & $\mathbf{0 . 6 5}$ & $\mathbf{0 . 4 2}$ & $\mathbf{0 . 4 8}$ & $\mathbf{0 . 4 1}$ & 0.19 & 0.31 & 0.14 & 0.15 \\
Dissolved oxygen & $\mathbf{0 . 7 0}$ & $\mathbf{0 . 4 5}$ & $\mathbf{0 . 7 3}$ & $\mathbf{0 . 6 6}$ & $\mathbf{0 . 4 8}$ & $\mathbf{0 . 4 8}$ & $\mathbf{0 . 3 5}$ & 0.04 \\
Redox potential & $\mathbf{0 . 6 0}$ & $\mathbf{0 . 5 7}$ & $\mathbf{0 . 7 2}$ & $\mathbf{0 . 8 1}$ & $\mathbf{0 . 5 0}$ & $\mathbf{0 . 6 8}$ & 0.09 & 0.11 \\
Salinity & 0.18 & 0.06 & 0.24 & 0.24 & -0.01 & $\mathbf{0 . 3 5}$ & 0.03 & 0.00 \\
Temperature & -0.61 & -0.24 & -0.64 & -0.26 & -0.34 & -0.15 & -0.31 & 0.11 \\
Sorting coefficient & -0.46 & -0.61 & -0.61 & -0.64 & -0.31 & -0.46 & 0.29 & -0.20 \\
Very coarse sand & 0.17 & 0.01 & 0.21 & $\mathbf{0 . 3 5}$ & 0.17 & $\mathbf{0 . 4 6}$ & -0.05 & -0.10 \\
Coarse sand & $\mathbf{0 . 4 8}$ & 0.25 & $\mathbf{0 . 5 5}$ & $\mathbf{0 . 5 3}$ & $\mathbf{0 . 3 6}$ & $\mathbf{0 . 4 9}$ & -0.07 & -0.08 \\
Medium sand & $\mathbf{0 . 5 3}$ & $\mathbf{0 . 5 4}$ & $\mathbf{0 . 6 4}$ & $\mathbf{0 . 7 4}$ & $\mathbf{0 . 4 4}$ & $\mathbf{0 . 6 4}$ & -0.03 & -0.02 \\
Fine sand & $\mathbf{0 . 5 7}$ & $\mathbf{0 . 6 5}$ & $\mathbf{0 . 5 6}$ & $\mathbf{0 . 6 2}$ & $\mathbf{0 . 3 5}$ & $\mathbf{0 . 4 9}$ & 0.18 & 0.21 \\
Very fine sand & 0.26 & 0.15 & $\mathbf{0 . 3 8}$ & 0.07 & 0.27 & 0.10 & 0.10 & 0.30 \\
Silt & -0.76 & $-\mathbf{0 . 7 0}$ & $\mathbf{- 0 . 8 3}$ & -0.88 & -0.55 & -0.76 & -0.12 & -0.12 \\
Clay & -0.68 & -0.65 & -0.79 & -0.88 & -0.52 & -0.80 & 0.01 & -0.18 \\
Total organic matter & -0.73 & -0.63 & -0.82 & -0.84 & -0.58 & -0.73 & -0.05 & -0.10 \\
Carbonates & $\mathbf{0 . 7 7}$ & 0.14 & $\mathbf{0 . 4 7}$ & 0.27 & 0.22 & $\mathbf{0 . 3 6}$ & 0.07 & -0.04 \\
\hline
\end{tabular}

Table 5. Trophic index (Ti) results for the five distinct trophic groups found in the three Guanabara Bay sectors in the dry and rainy seasons. Numbers in bold represent the two dominant trophic groups in each sector.

\begin{tabular}{lcccccc}
\hline & \multicolumn{3}{c}{ Dry Season } & \multicolumn{3}{c}{ Rainy Season } \\
\hline Feeding Guild & Inner & Middle & Outer & Inner & Middle & Outer \\
\hline Deposit feeder & $\mathbf{1 8 , 5 4}$ & $\mathbf{3 1 2 , 6 6}$ & $\mathbf{2 4 1 , 3 3}$ & $\mathbf{3 2 , 8 5}$ & $\mathbf{4 4 1 , 8 2}$ & $\mathbf{4 6 7 , 7 3}$ \\
Subsurface deposit feeder & - & 10,95 & 10,01 & - & 4,69 & 15,24 \\
Suspension feeder & 1,49 & 87,62 & 31,51 & 7,25 & 111,73 & 92,37 \\
Carnivore & 2,05 & $\mathbf{1 5 3 , 3 0}$ & $\mathbf{1 9 8 , 9 5}$ & $\mathbf{1 2 , 6 9}$ & $\mathbf{1 1 9 , 4 4}$ & $\mathbf{1 8 6 , 8 8}$ \\
Omnivore & $\mathbf{2 , 3 0}$ & 58,95 & 55,13 & 1,30 & 27,12 & 54,31 \\
\hline (-) absent & & & & &
\end{tabular}

\section{Classification of Benthic Assemblages}

The soft-bottom polychaete cluster groups responded similarly to the gradients of environmental variables analyzed. Excluding the azoic and impoverished stations, mostly located in the inner bay and that were excluded from this analysis, the softbottom polychaete fauna in the dry season clustered into three major groups (ANOSIM, $\mathrm{R}=0.784$; p<0.001) (Fig. 5). Group A was composed by 6 stations: 37, 36, 27, 28, 29 and 38. The SIMPER test (Table 6) revealed that group A was characterized by the carnivores Glycinde multidens, Nephtys squamosa and Goniada maculata, as well as omnivores such as Tharyx sp. and Kinbergonuphis tenuis. Group B included ten stations in the middle sector and one in the inner sector (Fig. 5). This group was characterized by those species that shared higher abundances at stations 22, 14, 15, 24 and 20 and by those sites with high diversities around the central channel (stations 23, 25 and 26). Four species were dominant or codominant in this cluster: Spiochaetopterus nonatoi, Prionospio heterobranchia, Goniada maculata and Poecilochaetus australis (SIMPER test, Table 6). Group C was composed by three stations (33, 34 and 35) represented by the deposit-feeder Prionospio heterobranchia, the suspension-feeder Chone insularis and the carnivore Eusyllis lameligera.

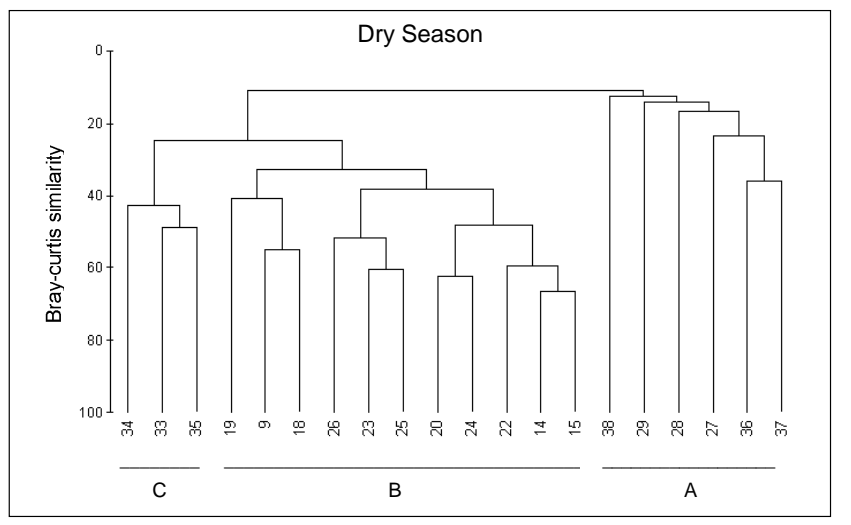

Fig. 5. Guanabara Bay Bray-Curtis clustering showing the three formed groups obtained in the ANOSIM analysis $(R=0,784)$ from polychaete abundance matrix generated for the dry season. Cluster numbers represent sampling stations. Stations with fewer than 6 individuals were excluded from the matrix. 
Table 6. SIMPER test results showing the mean similarity of the polychaete species clusters observed in the dry season, with the dominant taxa in each group. Cutoff $=70 \%$. For the mean dissimilarity only the percentage results are represented.

Dry Season

\begin{tabular}{|c|c|c|c|c|c|}
\hline Average similarity $=42.59 \%$ & Group B & & & & \\
\hline Species & Av.Abund & Av.Sim & Sim/SD & Contrib\% & Cum.\% \\
\hline Spiochaetopterus nonatoi & 29.45 & 13.51 & 1.89 & 31.71 & 31.71 \\
\hline Poecilochaetus australis & 154.82 & 7.46 & 0.82 & 17.51 & 49.22 \\
\hline Kinbergonuphis tenuis & 7.27 & 5.17 & 1.55 & 12.14 & 61.36 \\
\hline Paraprionospio pinnata & 8.73 & 4.60 & 1.12 & 10.81 & 72.16 \\
\hline Average similarity $=20.42 \%$ & Group A & & & & \\
\hline Species & Av.Abund & Av.Sim & Sim/SD & Contrib\% & Cum.\% \\
\hline Kinbergonuphis tenuis & 1.50 & 6.80 & 0.75 & 33.29 & 33.29 \\
\hline Nepthys squamosa & 3.00 & 5.66 & 0.66 & 27.72 & 61.01 \\
\hline Paraprionospio pinnata & 0.50 & 1.81 & 0.44 & 8.88 & 69.89 \\
\hline Ampharetidae & 0.50 & 1.81 & 0.44 & 8.88 & 78.77 \\
\hline Average similarity $=49.27 \%$ & Group C & & & & \\
\hline Species & Av.Abund & Av.Sim & Sim/SD & Contrib\% & Cum.\% \\
\hline Prionospio heterobranchia & 49.67 & 8.89 & 8.74 & 18.04 & 18.04 \\
\hline Eusyllis lamelligera & 30.67 & 6.35 & 10.09 & 12.88 & 30.92 \\
\hline Chone insularis & 41.67 & 4.29 & 5.00 & 8.72 & 39.64 \\
\hline Pseudoeurythoe ambigua & 10.00 & 2.08 & 2.70 & 4.23 & 43.87 \\
\hline Glycera americana & 2.67 & 2.05 & 3.46 & 4.17 & 48.04 \\
\hline Goniada maculata & 4.67 & 2.02 & 4.98 & 4.11 & 52.14 \\
\hline Aricidea sp. & 2.67 & 2.02 & 4.98 & 4.11 & 56.25 \\
\hline Neanthes sp. B & 2.67 & 2.02 & 4.98 & 4.11 & 60.36 \\
\hline Phyllodoce sp. & 1.33 & 1.72 & 11.13 & 3.49 & 63.85 \\
\hline Spiochaetopterus nonatoi & 1.67 & 1.72 & 11.13 & 3.49 & 67.34 \\
\hline Euclymene oerstedii & 5.67 & 1.57 & 0.58 & 3.18 & 70.52 \\
\hline \multicolumn{6}{|l|}{ Average dissimilarity: } \\
\hline \multicolumn{6}{|c|}{ Groups } \\
\hline$B \& A$ & $B \& C$ & \multicolumn{2}{|c|}{$A \& C$} & & \\
\hline $88.20 \%$ & $72.61 \%$ & \multicolumn{2}{|c|}{$89.73 \%$} & & \\
\hline
\end{tabular}

In the rainy season, only two groups were formed $(\mathrm{R}=0.887 ; \mathrm{p}<0.001)$ (Fig. 6). Group A comprised stations 36,37 and 38 , dominated by three species that occurred in low abundance: Nothria sp., Magelona riojai and the carnivore Nephtys squamosa (Table 7). Nine stations were added in the rainyseason cluster B, again represented by Spiochaetopterus nonatoi and Poecilochaetus australis, which occurred in high abundance. The former showed high abundances in a transition area from the middle to the inner sector, and the other, Poecilochaetus australis, almost duplicated its abundance at many sampling stations.

The SIMPER test revealed high dissimilarities between the groups, confirming the polychaete clustering results found. A ranking of the main polychaete species in the community structure configuration, and the average similarities and dissimilarities between the groups are listed for the dry (Table 6) and rainy seasons (Table 7). The BIOENV correlation revealed that oxygen saturation $\left(\mathrm{O}_{2} \%\right)$, redox potential (Eh) and the degree of grain sorting were the environmental variables that best explained the dry season polychaete spatial pattern $(\mathrm{R}=0.409)$. On the other hand, BIOENV test $(\mathrm{R}=0.442)$ confirmed the redox potential, salinity and the degree of sediment sorting as the main variables responsible for the polychaete community grouping in the rainy season.

Guanabara Bay can be divided into three main zones as follows: A) High polychaete diversity, hydrodynamically exposed areas composed of sands, oxidized or moderately reduced sediments with normoxy conditions in the water column. B) Low diversity and high biomass of deposit- and suspensionfeeding species in the middle part of the bay near continental influents, comprising stations sharing similar contributions of silt, clay and fine sands. C) Azoic area or an impoverished polychaete community in low-hydrodynamic energy areas of silt and clay with extremely reduced sediments, high total organicmatter content and hypoxic conditions in the water column (Fig. 7). 


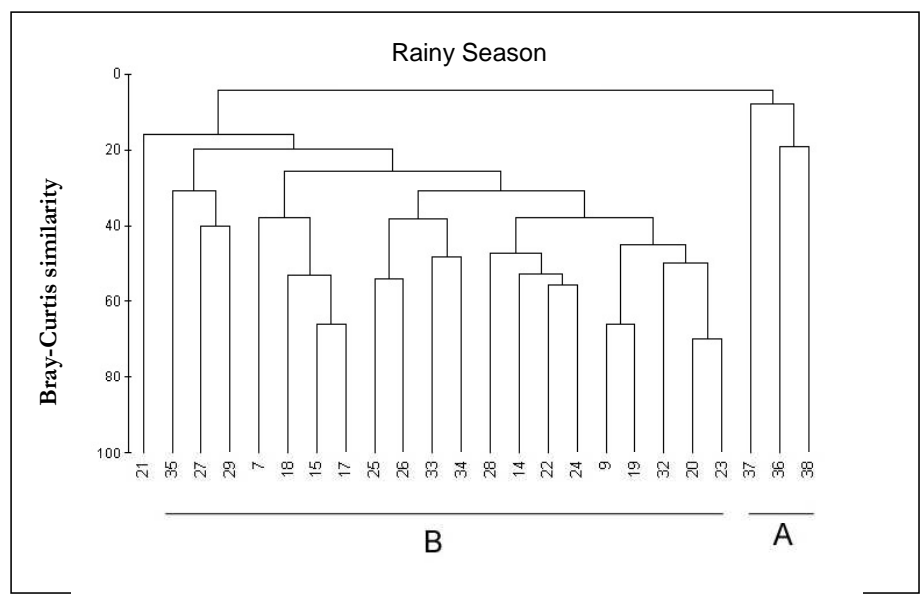

Fig. 6. Bray-Curtis clustering showing the three groups obtained in the ANOSIM analysis $(\mathrm{R}=0.887)$ of the polychaete abundance matrix recorded in the rainy season. Numbers represent sampling stations.

Table 7. SIMPER test results showing the mean similarity and dissimilarity of the polychaete species clusters observed in the rainy season, with the dominant taxa in each group. Cutoff $=70 \%$.

Rainy Season

\begin{tabular}{|c|c|c|c|c|c|}
\hline Average similarity $=31.95 \%$ & Group B & & & & \\
\hline Species & Av. Abund & Av. Sim & Sim/SD & Cum \% & \\
\hline Spiochaetopterus nonatoi & 65.76 & 17.28 & 1.14 & 54.09 & \\
\hline Poecilochaetus australis & 150.71 & 7.24 & 0.78 & 76.74 & \\
\hline Average similarity $=14.46 \%$ & Group A & & & & \\
\hline Species & Av. Abund & Av. Sim & $\mathrm{Sim} / \mathrm{SD}$ & Cum \% & \\
\hline Nothria sp. & 4.00 & 3.90 & 0.58 & 26.96 & \\
\hline Nephtys squamosa & 6.00 & 3.08 & 0.58 & 48.26 & \\
\hline Magelona riojai & 3.00 & 3.08 & 0.58 & 69.55 & \\
\hline Trypanosyllis parvidentata & 0.67 & 2.46 & 0.58 & 86.56 & \\
\hline Average dissimilarity $=95.94 \%$ & Group B & Group A & & & \\
\hline Species & Av. Abund & Av. Abund & Av. Diss & Diss/SD & Cum \% \\
\hline Spiochaetopterus nonatoi & 65.76 & 0.00 & 14.71 & 1.47 & 15.33 \\
\hline Poecilochaetus australis & 150.71 & 0.00 & 10.04 & 1.05 & 25.80 \\
\hline Nothria sp. & 0.29 & 4.00 & 6.50 & 0.81 & 32.57 \\
\hline Nepthys squamosa & 0.05 & 6.00 & 5.77 & 0.91 & 38.59 \\
\hline Magelona riojai & 0.00 & 3.00 & 4.63 & 1.00 & 43.41 \\
\hline Goniada maculata & 4.19 & 0.33 & 3.14 & 0.76 & 46.68 \\
\hline Eusyllis lamelligera & 2.71 & 2.00 & 2.95 & 0.77 & 49.75 \\
\hline Trypanosyllis parvidentata & 1.90 & 0.67 & 2.86 & 0.99 & 52.73 \\
\hline Onuphis eremita & 0.10 & 1.00 & 2.55 & 1.03 & 55.38 \\
\hline Eumida sanguinea & 2.71 & 0.00 & 2.54 & 0.71 & 58.03 \\
\hline Rhamphobrachium sp. & 0.29 & 0.67 & 2.46 & 0.63 & 60.59 \\
\hline Dispio uncinata & 0.05 & 1.00 & 2.17 & 0.64 & 62.85 \\
\hline Ninoe brasiliensis & 0.05 & 1.33 & 2.08 & 0.66 & 65.02 \\
\hline Paraprionospio sp. & 1.81 & 0.00 & 2.02 & 0.68 & 67.13 \\
\hline Prionospio heterobranchia & 9.52 & 0.00 & 1.99 & 0.66 & 69.20 \\
\hline Tharyx sp. & 1.29 & 0.33 & 1.99 & 0.80 & 71.27 \\
\hline
\end{tabular}




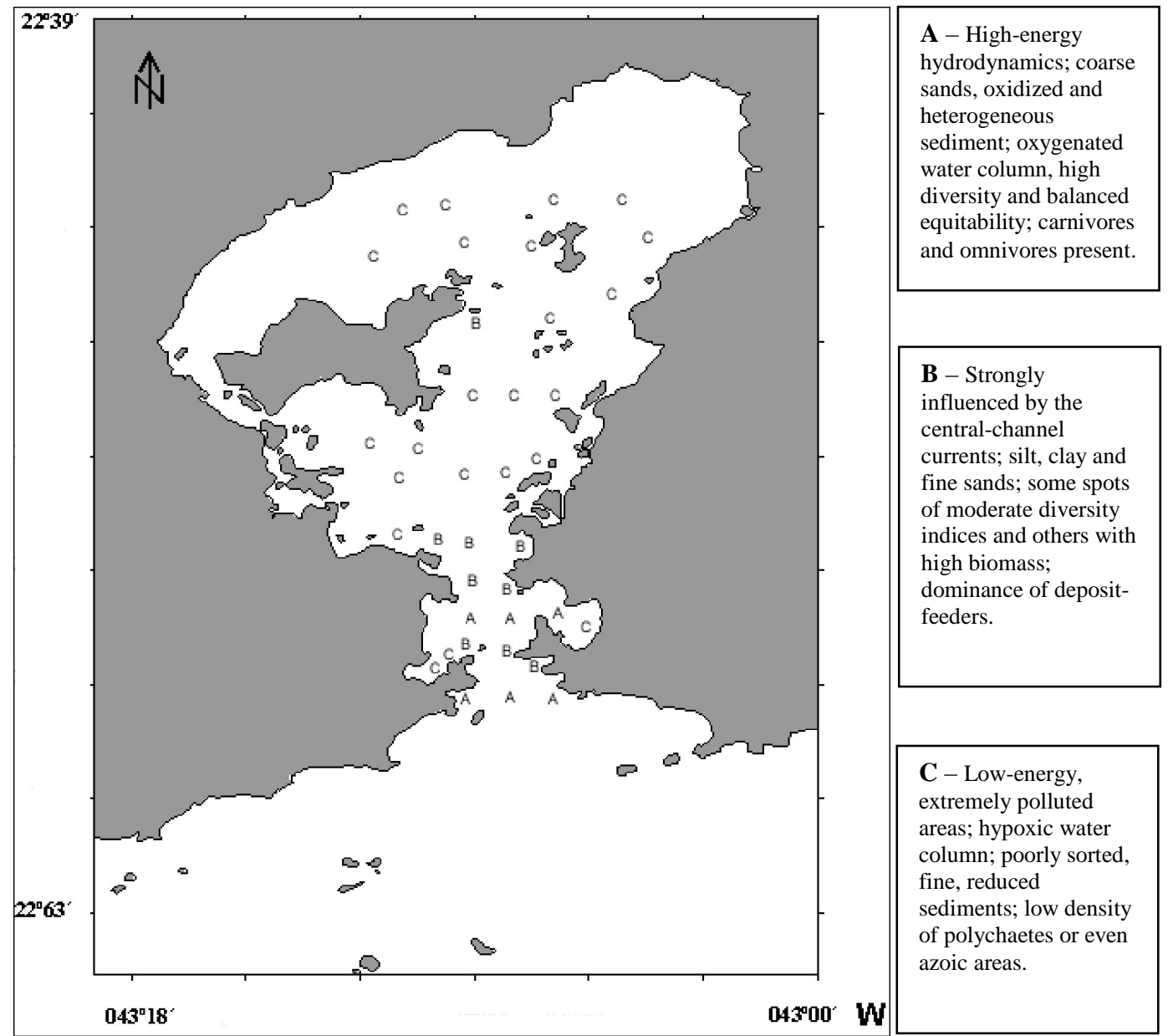

Fig. 7. Soft-bottom polychaete fauna and environmental characteristics for the three faunistic zones in Guanabara Bay.

\section{Discussion}

The soft-bottom polychaete community of Guanabara Bay consists of several unique assemblages distributed throughout different regions of the bay. Most of the animals composing these assemblages belong to a relatively small number of species, which reflects the unstable habitat typical of many embayments. Hydrodynamic conditions appeared to be the primary factor influencing the distribution of polychaete assemblages in Guanabara Bay. Anthropogenic impact may represent a secondary factor that influenced the distribution of the sublittoral polychaeta infauna.

Polychaete diversity increases from the azoic area and impoverished stations in the inner sector and enclosing areas, to a well-structured community in terms of species composition, high evenness and mixed trophic groups inhabiting the outer sector of the bay. An evident environmental gradient was observed from the oceanic zone towards the inner parts of the bay, probably due to the water circulation pattern and environmental conditions.

Polychaete species richness and diversity increased towards the mouth of the bay. This can be explained in part by a more heterogeneous granulometry in the outer parts of the bay compared with the inner areas. The low diversity recorded in the inner bay could be a result of the tendency to habitat monotony (silt and clay) and of high levels of total organic matter trapped in these areas. Similar patterns relative to hydrodynamic gradients have been reported previously, and are typical of estuarine benthic communities (SUMICH, 1992; DEXTER; CROOKS, 2000). NALESSO et al. (2005) found a similar pattern in the soft-bottom macrobenthic communities of Vitória Bay in southeastern Brazil, where species 
richness, Shannon-diversity and total abundance decrease from outer-bay stations towards the inner bay. In estuarine communities, diversity is often influenced by the natural variability of the system (SAIZ-SALINAS; GONZÁLEZ-OREJA, 2000). Diversity is usually lowest in physically extreme environments (SNELGROVE; BUTMAN, 1994) and eutrophic areas (PEARSON; ROSENBERG, 1978). Most of the soft-bottom areas studied in Guanabara Bay are under eutrophic conditions, with extremely reduced sediment and high concentrations of total organic matter. The more heterogeneous and complex the physical environment becomes, the more complex and diverse are the animal communities supported by a certain environment (PIANKA, 1966). Although we did not measure any physical oceanographic data in the present study, we presume that the hydrodynamic regime influenced the polychaete distribution, building new habitats, dispersing polychaete larval stages throughout the bay, and influencing recruitment success. The soft-bottom environment and polychaete fauna of the outer part of the Bay is probably influenced by those features.

Some observations in the present study suggest that the sublittoral polychaete infauna was also affected by high amounts of organic load coming from urban areas. The bay showed a clear gradient of severe eutrophication (inner sector) to less-eutrophic conditions farther from the continental margin (outer sector), explained herein by the total organic matter measurements, high primary production in the water column and reduced sediments recorded in the inner sector. Species richness is usually low in regions of the bay that are impacted by anthropogenic factors (KENNISH, 1997). Anthropogenic impacts usually reduce the occurrence of rare species in a certain area (MAY, 1981). COGNETTI (1978) noted that pollution simplifies the spatial-temporal polychaete species distribution, with a progressive decrease in the number of species, except those able to survive in severe conditions. This biological pattern seems to be present at the Guanabara Bay sites, which have some of the highest concentrations of organic and inorganic loads of all coastal bays in the world (CARREIRA et al., 2002; PERIN et al., 1997). The dominance of a few species towards the inner parts of the bay, such as Prionospio heterobranchia, Spiochaetopterus nonatoi and Poecilochaetus australis, demonstrates that these species are probably indicators of organic enrichment. The monotonous habitat in the inner parts of the bay may also have contributed to favoring a small number of eurytopic species.

Although no sedimentation measurements were made in the present study, the continental runoff is likely higher during the rainy season, increasing the amount of fine sediments and pollutants that reach the inner bay. This condition may change the soft-bottom habitat to some extent, and thus the biological processes. Other studies in the bay have recorded that the mean rainfall during a 30-year period (1961-1990) varied from $291 \mathrm{~mm} \mathrm{month}{ }^{-1}$ in the mountains to a minimum of $30 \mathrm{~mm} \mathrm{month}^{-1}$ in the winter in low-lying areas (KJERFVE et al., 1997). During the austral summer, heavy rains may exceed $10 \mathrm{~mm}$ in a couple of hours, producing intense runoff and flooding of low-lying fringe areas (ALCÂNTARA; WASHINGTON, 1989). Annually, the mean runoff entering the bay is approximately $351.48 \mathrm{~m}^{3} \mathrm{~s}^{-1}$ oscillating between $166.8 \mathrm{~m}^{3} \mathrm{~s}^{-1}$ in winter and 551.7 $\mathrm{m}^{3} \mathrm{~s}^{-1}$ in summer (JICA, 1994). The estimated total annual solid discharge and solid discharges of tributary rivers are about 4 million tons year ${ }^{-1}$ (HIDROSERVICE-GEIPOT, 1974). As pointed out by DATTA and SARANGI (1987), the pressure imposed by salinity, turbidity, dissolved oxygen and organic matter input to the estuaries appears to have a direct negative effect on abundance and diversity during periods of heavy monsoon rains. The temporal species exclusion and dominance of fewer species in the rainy period could be influenced by input of fine fractions, increasing the sedimentation rates and the amount of pollutants found in the soft bottoms during this season.

The high diversity found in some areas around the central channel of the bay may be linked with the increased organic-matter content combined with the better water renewal in the main channel, which reduce the deleterious effects of organic enrichment. GRAY et al. (2002) stated that the major effects on benthic fauna result more from hypoxia than from organic enrichment. In fact, sewage discharge alone did not severely affect the diversity patterns in a small Norwegian sill system (JØRGENSEN, 1980; HOLTE, 2001). Furthermore, any increase in the influx of organic material that does not reduce permeability, provides additional food for the organisms, increasing the psammic biomass and thereby the capacity of the sand deposit for processing even large quantities of organics (WEBB, 1991). KJERFVE et al. (1997) reported that the time to replace $50 \%$ of the water volume of Guanabara Bay is 11.4 days, which is considered fast compared with other bays. This may explain in part why in some parts of the bay there is relatively good water quality in spite of large quantities of untreated sewage input. Méndez and Romero (1998) found a similar pattern of soft-bottom polychaete communities in a pollution gradient off Barcelona, with highest diversity values at sites not far from the sewage outfall, where moderate hydrodynamic energy is combined with coarse sediment and considerable organic-matter input.

The low diversity found in the inner bay is probably due to anoxic conditions of the sediment caused by the stagnant and limited water exchange associated with nutrient discharges, either directly as 
sewage or indirectly from continental runoff. The polychaete fauna probably did not tolerate the high amounts of organic matter and hypoxic conditions in the inner sector and in some stations of the middle sector recorded in the present study. There are strong indications that increases in carbon storage occurred in response to growing eutrophication conditions. In spite of the high respiration rates occurring in the water column, rapid sedimentation rates result in the transfer of a significant carbon fraction to anoxic sediments (CARREIRA et al., 2002). Higher mean concentrations of nutrients are found near the inner western shore, due to enrichment from sewage runoff and a low rate of water renewal of the inner parts of the bay. MARCOMINI et al. (1995) reported that three decades of changes in the physical and chemical characteristics of the water, sediment and hydrodynamic conditions were followed by a marked change in the benthic community, especially in less hydrodynamic areas. The Guanabara Bay soft-bottom polychaete community responds to this predicted pattern, as all of the azoic stations were recorded in locations under intense siltation, weak hydrodynamic energy and low dissolved-oxygen concentration, i.e., in the inner sector.

The polychaete trophic structure in Guanabara Bay followed the general pattern expected for an estuary characterized by great habitat heterogeneity. The dominance of deposit feeders in the inner sectors, suspension feeders, deposit feeders and carnivores in some stations of the middle sector, and the mixed contribution of all the trophic groups in the outer sector concord with the sublittoral trophic spatial pattern recorded in other studies (PAIVA, 1993; PINEDO et al., 1997; MUNIZ; PIRES, 1999). In general, coarse-grained sediment supported the highest densities of suspension-feeders (RHOADS; YOUNG, 1970; GRAY, 1974), whereas the silt-clay area was dominated by deposit-feeders (SANDERS, 1968). The dominance of deposit-feeders in both periods, but more intensely in rainy periods, may be linked to the soft-bottom composition of the study area (silt and clay) and the input of organic matter, providing a continuous source of food for deposit-feeding polychaetes throughout the year. Studying a temperate estuary, Lopez and Levinton (1987) stressed that the response of deposit-feeders to seasonal input of organic matter suggests that they are food-limited during much of the year. Moreover, in such areas the increases in deposit-feeding benthos appeared to be a response to seasonal deposition of the early-spring diatom bloom (RUDNICK, 1985). Datta and Sarangi (1987) showed that tropical sublittoral infaunas respond negatively to freshwater inputs during monsoons, but recolonize quickly and are dominated by small surface deposit-feeding polychaetes as a result of continuous input of organic matter to the bottom layers (ALONGI, 1989). Spionidae and Poecilochaetidae were the two most important polychaete families in the deposit-feeder category. Spionids have long grooved palps that are useful to capture food particles in the bottom layer and in the water-sediment interface (FAUCHALD; JUMARS, 1979). The dominance of spionids might be related in part to the competitive success in obtaining food in favorable habitat conditions (high amounts of total organic matter and fine sediment fractions, silt and clay, good for tube building) compared with other polychaete families recorded in the study area.

No direct evidence could be obtained with this sampling design that could confirm the direct effect of pollutants on the polychaete infauna. Moreover, the lack of historical data on the polychaete infauna in the area made further conclusions impossible based on the data from the two sampling periods of this study. Additional studies using key species (e.g., Poecilochaetus australis and Spiochaetopterus nonatoi) present in the area are necessary to clarify the true impact of organic pollutants on the local biota.

\section{ACKNOWLEDGEMENTS}

We express our thanks to the FAPERJ research agency for providing financial support of part of this project. We are also very grateful to MSc. Joel Braga de Mendonça Júnior for his help in fieldwork, and to Dr. Elianne P. Omena for polychaete species identification. Special thanks to Mr. Jean-Michel Amouroux (Observatoire Océanologique Banyuls-surMer, France), Natalia Venturini (IOUSP) and Andrea Junqueira (UFRJ) for important comments, criticisms and ideas. MT thanks the CNPq (National Council for the Development of Science and Technology, Brasília) for supporting studies on biodiversity in the form of ongoing grants 302065/2007-5 and PETROBRAS for financial support (process number 4600224970).

\section{REFERENCES}

ALONGI, D. M. Ecology of tropical soft-bottom benthos: a review with emphasis on emerging concepts. Revta Biol. trop., v. 37, n. 1, p. 85-100, 1989.

ALCÂNTARA, F.; WASHINGTON, D. C. An analytical synoptic-dynamic study about the severe weather event over the city of Rio de Janeiro on Jan 2, 1987: In, MAGOON, O.; NEVES, C. (Ed.). Coastlines of Brazil, New York, NY: American Society of Civil Engineers, 1989. p. 195-204.

AMADOR, E. S. Baía de Guanabara e ecossistemas periféricos - Homem e Natureza. Published by the author, 1997. p. 539.

ANDRADE, L.; GONZALEZ, A. M.; ARAÚJO, F. V.; PARANHOS, R. Flow cytometry assessment of bacterioplankton in tropical marine environments. J. Microbiol. Meth., The Netherlands, v. 55, n. 3, p. 841850, 2003. 
ANDRADE, D. S. D.; MACIEL, N. C. Os manguezais do recôncavo da Baía de Guanabara. Cadernos FEEMA, série técnica 10/79. Rio de Janeiro, 1979. p. 113.

CARREIRA, R. S.; WAGENER, A. L. R.; READMAN, J. FILEMAN, M. S. A.; VEIGA, A. Changes in the sedimentary organic carbon pool of a fertilized tropical estuary, Guanabara Bay, Brazil: an elemental, isotopic and molecular marker approach. Mar. Chem., v. 79, p. 207-227, 2002.

CLARKE, K. R.; WARWICK, R. M. Change in marine communities: an approach to statistical analysis and interpretation. Plymouth: Plymouth Marine Laboratory, 1994. p. 144.

COGNETTI, G. On some aspects of the ecology of the benthic littoral Polychaetes. Boll. Zool., v. 45, p. 145 $154,1978$.

DATTA, N. C.; SARANGI, N. Benthic macroinvertebrate community of estuarine waters of west Bengal, India. In: THOMPSON, M. F.; SAROJINI, R.; BUHUSHANAM, R. N. (Ed.). Biology of benthic marine organisms. Techniques and methods as applied to the Indian Ocean. Rotterdam, The Netherlands, 1987. p. 247-255.

DEXTER, D. M.; CROOKS, J. A. Benthic communities and the invasion of an exotic mussel in Mission Bay, San Diego: A long-term history. Bull. South. Calif. Acad. Sci., v. 99, n. 3, p. 128-146, 2000.

FARACO, L. F. D; LANA, P. C. Response of polychaetes to oil spills in natural and defaunated subtropical mangrove sediments from Paranaguá Bay (SE Brazil). Hydrobiologia, v. 496, n. 1-3, p. 321-328, 2003

FAUCHALD, K.; JUMARS, P. A. The diet of worms: a study of polychaete feeding guilds. Oceanogr. mar. Biol. ann. Rev., v. 1, p. 193-284, 1979.

FLORES, JR., R. G.; BARRETO M. K.; COSTA, H. R. On the relationship between ecological and physicochemical water quality parameters: a case study at the Guanabara Bay, Rio de Janeiro. In: Ecological diversity in theory and practice. Fairland, Maryland: International Co-operative Publishing House, 1979. p. 195-206.

GRASSHOFF, K.; EHRHARDT, M.; KREMLIING, K. Methods of sea water analysis. 2nd ed. Weinheim: Verlag Chemie, 1983. 419 p.

GRAY, J. S. Animal-sediment relationships. Oceanogr. mar. Biol. ann. Rev., v. 12, p. 223-261, 1974.

GRAY, J. S. Species richness of marine soft sediments. Mar. Ecol. Progr. Ser., v. 244, p. 285-297, 2002.

GRAY, J. S.; WU, R. S.; OR, Y. Y. Effects of hypoxia and organic enrichment on the coastal marine environment. Mar. Ecol. Prog. Ser., v. 238, p. 249-279. 2002.

GUILLE, A. Benthic bionomy of continental shelf of the French Catalane coast. II. Benthic communities of the macrofauna. Vie Milieu, v. 21, n. 8, p. 149-280, 1970.

HIDROSERVICE-GEIPOT. Hidroservice Engenharia de Projetos e Grupo de Estudos para a Integração Política de Transporte (GEIPOT) - Prestação de serviços de consultoria para a elaboração de plano diretor para aproveitamento da área do contorno do fundo da Baía de Guanabara. Rio de Janeiro. Relatório final, 1974.

HOLTE, B. Possible ecological effects from Maldanid (Annelida, Polychaeta) "super-dominance" in a small North Norwegian sill system. Ophelia, v. 55, n. 1, p. 6975. 2001 .
JICA. The study on recuperation of the Guanabara Bay Ecosystem. Main Report, vol. 2. Tokyo: Japan International Cooperation Agency, Kokusai Kogyo Co., Ltd, 1994.

JONES, G. P.; KALY, U. L. Criteria for selecting marine organisms in biomonitoring studies. In: SCHMITT, R.J.; ROSENBERG, C.W., (Ed.). Detecting ecological impacts - concepts and applications in coastal habitats. San Diego: Academic Press, 1996. p. 29-45.

JØRGENSEN, B. B. Seasonal oxygen depletion in the bottom waters of a Danish fjord and its effects on the benthic community. Oikos, v. 34, p. 68-76, 1980.

KENNISH, M. J. Pollution impacts on marine biotic communities. Boca Raton, Florida: CRC Press, 1997. 310 p.

KJERFVE, B.; RIBEIRO, C. H. A.; DIAS, G. T. M.; FILIPPO, A. M.; QUARESMA, V. S. Oceanographic characteristics of an impacted coastal bay: Baía de Guanabara, Rio de Janeiro, Brazil. Cont. Shelf Res., v. 17, n. 13, p. 1609-1643, 1997.

LOPEZ, G. R.; LEVINTON, J. S. Ecology of depositfeeding animals in marine sediments. Q. Rev. Biol., v. 62, n. 3, p. 235-260, 1987.

LU, L.; GOH, B. P. L.; CHOU, L. M. Effects of coastal reclamation on riverine macrobenthic infauna (Sungei Punggol) in Singapore. J. aquat. Ecosyst. Stress Recov., v. 9, p. 127-135, 2002.

MARCOMINI, A.; PAVONI, B.; ORIO, A.A. Eutrophication of the Lagoon of Venice: Nutrient loads and exchanges. In: Eutrophic shallow estuaries and lagoons. Murdoch: Institute for Environmental Science, Murdoch University. p. 59-80, 1995.

MARTIN, D.; BALLESTEROS, E.; GILI, J. M.; PALACÍN, C. Small-scale structure of infaunal Polychaete communities in an estuarine environment: Methodological Approach. Estuar. coast. Shelf Sci., v. 36, p. 47-58, 1993.

MAY, R. M. Patterns in multi-species communities. In: MAY, R. (Ed.). Theoretical ecology: Principles and applications. Blackwell. p. 197-227, 1981.

MAYR, L.M.; TENENBAUM, D.R.; VILLAC, M.C.; PARANHOS, R.; NOGUEIRA, R.; BONECKER, S.L.C.; BONECKER, A.C. Hydrobiological characterization of Guanabara Bay. In: MAGOON, O.T.; NEVES, O. (Ed.). Coastlines of Brazil. American Society of Civil Engineers, New York, 1989. p. 124-139.

MENDES, C.L.T.; TAVARES, M.; SOARES-GOMES, A. Taxonomic sufficiency for soft-bottom sublittoral mollusks assemblages in a tropical estuary, Guanabara Bay, Southeast Brazil. Mar. Pollut. Bull., v. 54, p. 377384, 2007.

MÉNDEZ, N.F.J.; ROMERO, J. Littoral soft-bottom polychaete communities in a pollution gradient in front of Barcelona (Western Mediterranean, Spain). Bull. mar. Sci., v. 63, n. 1, p. 167-178, 1998.

MUNIZ, P.; PIRES, A.M.S. Trophic structure of polychaetes in the São Sebastião Channel (southern Brazil). Mar. Biol., v. 134, p. 517-528, 1999.

MUNIZ, P.; PIRES, A.M.S. Polychaete association in a subtropical Environment (São Sebastião Channel, Brazil): a structural analysis. Mar. Ecol., v. 21, n. 2, p. $145-160,2000$ 
NALESSO, R. C., JOYEUX, J-C.; QUINTANA, C. O.; TOREZANI, E.; OTEGUI, A.C.P. Soft-bottom macrobenthic communities of the Vitória Bay Estuarine System, South-Eastern Brazil. Braz. J. Oceanogr., v. 53, n. 1/2, p. 23-38, 2005.

OLIVEIRA, L. P. H. Poluição das águas marítimas, estragos na flora e fauna do Rio de Janeiro. Mem. Inst. Oswaldo Cruz, v. 56, p. 39-59, 1958.

OLIVEIRA, L.P.H.; KRAU, L. Estudos aplicados à recuperação biológica da Baía de Guanabara. Mem. Inst. Oswaldo Cruz, v. 74, n. 2, p. 99-145, 1976.

PAIVA, P.C. Trophic structure of a shelf polychaete taxocoenosis in southern Brazil. Cah. Biol. mar., v. 35, p. 39-55, 1993.

PARANHOS, R.; MAYR, L.M. Seasonal patterns of temperature and salinity in Guanabara Bay, Brazil. Fresenius Environ. Bull., v. 2, p. 647-652, 1993.

PARANHOS, R.; NASCIMENTO, S. M.; MAYR, L. M. On the faecal pollution in Guanabara Bay, Brazil. Fresenius Environ. Bull., v. 4, p. 352-357, 1995.

PEARSON, T. H.; ROSENBERG, R. Macrobenthic succession in relation to organic enrichment and pollution of the marine environment. Oceanogr. mar. Biol. ann. Rev., v. 16, p. 229-311, 1978.

PERIN, G.; FABRIS, R.; MANENTE, S.; WAGENER, A.R.; HAMACHER, C.; SCOTTO, S. A five-year study of the heavy-metal pollution of Guanabara Bay sediments (Rio de Janeiro, Brazil) and evaluation of the metal bioavailability by means of geochemical speciation. Wat. Res., v. 31, n. 2, p. 3017-3028, 1997.

PIANKA, E. R. 1966. Latitudinal gradients in species diversity: a review of concepts. Am. Naturalist, v. 100, n. 910, p. 33-46, 1966.

PINEDO, S.; SARDÁ, R.; MARTIN, D. Comparative study of the trophic structure of soft-bottom assemblages in the Bay of Blanes (western Mediterranean Sea). Bull. mar. Sci., v. 60, n. 2, p. 529-542, 1997.

POCKLINGTON, P.; WELLS, P.G. Polychaetes. Key taxa for marine environmental quality monitoring. Mar. Pollut. Bull., v. 24, n. 12, p. 593-598, 1992.

REBELLO, A.L.; PONCIANO, C.R.; MELGES, L.H. Avaliação da produtividade primária e da disponibilidade de nutrientes na Baía de Guanabara. An. Acad. bras. Ciênc., v. 60, p. 419-430, 1988.

RHOADS, D.C.; YOUNG, D.K. The influence of depositfeeding organisms on sediment stability and community trophic structure. J. mar. Res., v. 28, n. 2, p. 150-178, 1970.

ROUSE, G.W; FAUCHALD, K. Cladistics and polychaetes. Zool. Scr., v. 26, p. 139-204.

RUDNICK， D.T.R.; ELMGREN， R.; FRITHSEN，J.B Meiofaunal prominence and benthic seasonality in a coastal marine ecosystem. Oecologia (Berl.), v. 67, p. 157-168, 1985.
SAIZ-SALINAS, J.I.; GONZÁLEZ-OREJA, J.A. Stress in estuarine communities: lessons from the highly-impacted Bilbao estuary (Spain). J. aq. Ecos. Stress Recov., v. 7, p. 43-55, 2000.

SANDERS, H.L. Marine benthic diversity: a comparative study. Am. Naturalist, v. 102, n. 925, p. 243-282, 1968.

SILVA, C.S.; RODRIGUES, J.C.V.; CÂMARA, N.L. Saneamento básico e problemas ambientais na região metropolitana do Rio de Janeiro. Revta bras. Geogr., n. 52, p. 5-106, 1980.

SILVA, L.F.B.; TAVARES, M.; SOARES-GOMES, A. Taxonomic sufficiency for soft-bottom sublittoral mollusks assemblages in a tropical estuary, Guanabara Bay, Southeast Brazil. Revta bras. Zool., v. 25, n. 4, p. 617-623, 2008.

SNELGROVE, P.V.R.; BUTMAN, C. A. Animal-sediment relationships revisited: cause versus effect. Oceanogr. mar. Biol. ann. Rev., v. 32, p. 111-177, 1994.

STRICKLAND, J.D.H.; PARSONS, T.R. A practical handbook of sea water analysis. Bull. Fish. Res. Bd Can., 1968. 311 p.

SUGUIO, K. Introdução à sedimentologia. São Paulo, Edgard Blücher, 1973. $317 \mathrm{p}$.

SUMICH, J. L. An introduction to the biology of marine life. Dubuque, Iowa: Wm. C. Brown Publishers, 1992. $213 \mathrm{p}$.

VAN DER VEM, M.; SOARES-GOMES, A.; TAVARES, M. Taxocene of Crustacea at a Highly Impacted Bay: Guanabara Bay, Southeastern Brazil. J. coast. Res., v. 39, p. 1135-1139, 2006.

VENTURINI, N.; TOMMASI, L. R.; BÍCEGO, M. C. ; MARTINS, C. C. Characterization of the benthic environment of a coastal area adjacent to an oil refinery, Todos os Santos Bay (NE-Brazil). Braz. J. Oceanogr., v. 52, p. 123-134, 2004.

VERGARA FILHO, W.; ALVES, J.R.P.; MACIEL, N.C. Diversity and distribution of crabs (Crustacea, Decapoda, Brachyura) in mangroves of the Guanabara Bay, Rio de Janeiro, Brazil, In: KJERFVE, B.; LACERDA, L.D.; DIOP, E.H.S. (Eds.) Mangrove ecosystem studies in Latin America and Africa. Paris: UNESCO, 1997. p. 155-162.

WEBB, J.E. Hydrodynamics, organisms and pollution of coastal sands. Ocean Shoreline Mgmt, v. 16, p. 23-51, 1991.

WIDBOM, B. Determination of average individual dry weights and ash-free dry weights in different sieve fractions of marine meiofauna. Mar. Biol., v. 84, p. 101108, 1984.

(Manuscript received 31 July 2008; revised 27 February 2009; accepted 24 June 2009) 